\title{
Emergent electromagnetism in solids
}

\author{
Naoto Nagaosa ${ }^{1,2 *}$ and Yoshinori Tokura ${ }^{1,2}$ 计 \\ 1 Department of Applied Physics, \\ University of Tokyo, 7-3-1, Hongo, \\ Bunkyo-ku, Tokyo 113-8656, Japan \\ ${ }^{2}$ Cross-Correlated Materials Research Group (CMRG), \\ and Correlated Electron Research Group (CERG), \\ RIKEN-ASI, Wako, Saitama 351-0198, Japan
}

(Dated: September 27, 2011)

\begin{abstract}
Electromagnetic field (EMF) is the most fundamental field in condensed-matter physics. Interaction between electrons, electron-ion interaction, and ion-ion interaction are all of the electromagnetic origin, while the other 3 fundamental forces, i.e., gravitational force, weak and strong interactions are irrelevant in the energy/length scales of condensed-matter physics. Also the physical properties of condensed-matters such as transport, optical, magnetic and dielectric properties, are almost described as their electromagnetic responses. In addition to this EMF in the low energy sector, it often happens that the gauge fields appear as the emergent phenomenon due to the projection of the electronic wavefunctions onto the curved manifold of the Hilbert sub-space. These emergent electromagnetic fields (EEMF's) play important roles in many places in condensed-matter physics including the quantum Hall effect, strongly correlated electrons, and also in non-interacting electron systems. In this article, we describe its fundamental idea and some of the applications recently studied.
\end{abstract}

PACS numbers: 73.43.Cd,72.25.-b,72.80.-r

\section{INTRODUCTION AND BASIC CONCEPTS}

Electromagnetism is the first gauge theory which was recognized in physics, and essentially contains the principle of the relativity. Most of the physical properties of solids are regarded as the responses of the systems to the electromagnetic field (EMF). For example, the transport phenomena are described by the currents induced by the electric field, magnetism is the behavior of the magnetization in response to the magnetic field, and optical properties are described by the electric polarization driven by the EMF of light. On the side of the electronic system, the electron-electron and/or electron-ion interactions mediated by the EMF, i.e., mostly the Coulomb interaction, determine the quantum state even without the external EMF as a probe. This interaction often leads to the emergent electromagnetic fields (EEMF's) as will be described in more details below. The other aspect of the electromagnetism in solids is the various cross correlation effect. Namely various different combinations of the inputs and responses have been explored. For example, an applied magnetic field can induce the electric polarization, or the magnetization emerges under the electric field in the magneto-electric (ME) effect. This effect was proposed long time ago 1], but has been very weak. An explosion of the research was triggered by the discovery of the multiferroic materials showing both the magnetic order and ferroelectricity which are coupled strongly to

\footnotetext{
*Electronic address: nagaosa@ap.t.u-tokyo.ac.jp
}

${ }^{\dagger}$ Electronic address: tokura@ap.t.u-tokyo.ac.jp each other [2, 3]. These phenomena are driven by the internal degrees of freedom of electrons, i.e., spin, charge and orbital, which are again described coherently by the EEMF's.

This EEMF is analogous to the usual EMF, but is much richer because of the following aspects [4, 5]. (i) It is defined on the lattice because of the crystal structure and Bloch waves in solids, and the lattice gauge theories are relevant. Therefore, various topological defects play important roles. (ii) One can generalize the space where EEMF is defined including the real space, momentum (k-)space, and other parameter space. (iii) The gauge group is not only $\mathrm{U}(1)$ as in the case of EMF, but can be more general such as the $\mathrm{SU}(2)$ corresponding to the Kramer's doublet in the time-reversal symmetric system, and $\mathrm{U}(\mathrm{N})$ corresponding to the N-bands system. (iv) Topological terms such as Chern-Simons term, WZW term, and $\theta$-term often appear to the effective action for EEMF. Therefore, solids can be an ideal arena to study various concepts and techniques in quantum field theory [].

The basic reason why the EEMF's appear in condensed-matter is the restriction of the Hilbert space. Namely, only the small portion of the Hilbert space is relevant to the low energy phenomena, which constitutes the fiber-bundle structure. For example, consider a vector moving on the surface of unit sphere $S^{2}$. The parallel transport of this vector on $S^{2}$ leads to the change in its direction as shown in Fig.1. This is because $S^{2}$ has the (constant) curvature and the vector picks up this curvature through the connection for infinitesimal parallel transport. This connection corresponds to the vector potential of EMF while the curvature to the magnetic 
flux, which are related via Stokes theorem to each other. An analogy can be drawn between this geometry problem and the quantum mechanics [7]. The vector corresponds to the wavefunction and $S^{2}$ to the restricted Hilbert space. Therefore, the parallel transport on the subspace leads naturally to the gauge structure. We discuss below briefly the Berry phase [8] and the spin-orbit interaction from this viewpoint.

\section{A. Berry phase of electrons in perfect crystal}

The Bragg diffraction of the electrons by the periodic electric field of the ions in a crystal leads to the Bloch states as described by

$$
\psi_{n \mathbf{k} \sigma}(\mathbf{r}, s)=e^{i \mathbf{k} \cdot \mathbf{r}} u_{n \mathbf{k}}(\mathbf{r}) \chi_{\sigma}(s),
$$

where $u_{n \mathbf{k}}(\mathbf{r})$ is the periodic function with respect to the translation of lattice vectors, $\chi_{\sigma}(s)$ is the spin wavefunction for $\sigma=\uparrow, \downarrow$ with the spin coordinate $s= \pm$. The band index $n$ and the crystal momentum $\mathbf{k}$ are the good quantum numbers and the energy eigenvalues $\varepsilon_{n}(\mathbf{k})$ 's with different $n$ 's are usually separated by the band gaps. This means that the electrons in a solid are confined to each band in the lower energy sector than the band gaps, which corresponds to the projection of the wavefunctions to the sub-space of the Hilbert space. This sub-space is usually "curved" and is represented by the gauge connection. (This is an application of the Berry connection to Bloch electrons [8].) It is given by the expression

$$
a_{n a}(\mathbf{k})=-i\left\langle u_{n \mathbf{k}}\left|\partial_{k_{a}}\right| u_{n \mathbf{k}}\right\rangle
$$

with $a=x, y, z$. This quantity appears in the innerproduct of the 2 wavefunctions for the 2 neighboring $\mathbf{k}$ points as

$$
\left\langle u_{n \mathbf{k}} \mid u_{n \mathbf{k}+\Delta \mathbf{k}}\right\rangle=\exp \left(i \mathbf{a}_{n}(\mathbf{k}) \cdot \Delta \mathbf{k}\right) .
$$

This Berry connection leads to the concept of Berry curvature corresponding to the magnetic field in the momentum space as

$$
b_{n a}(\mathbf{k})=\varepsilon_{a b c} \partial_{k_{b}} a_{n c}(\mathbf{k})
$$

with the totally antisymmetric tensor $\varepsilon_{a b c}$.

What is the physical consequences of this Berry phase ? To answer to this question, it is useful to consider the motion of a wave-packet made of the Bloch states, where the position and momentum of a particle is defined within the accuracy consistent with the uncertainty principle [9]. In the presence of the Berry connection, the position operator $\mathbf{x}$ originally defined as $x_{\mu}=i \partial / \partial k_{\mu}$ should be generalized to the gauge covariant form $x_{\mu}=i \partial / \partial k_{\mu}+a_{n \mu}(k)$. This is dual to the momentum operator $\pi_{\mu}$ in the presence of the magnetic field, i.e., $\pi_{\mu}=p_{\mu}+e A_{\mu}(\mathbf{x})=-i \partial / \partial x_{\mu}+e A_{\mu}(\mathbf{x})$, where $A_{\mu}(\mathbf{x})$ is the vector potential of the EMF. Analogously to the commutator $\left[\pi_{x}, \pi_{y}\right]=i e B_{z}(\mathbf{x})$ with $B_{z}(\mathbf{x})$ being the $z$-component of the magnetic field, one can derive $[x, y]=i b_{z}(\mathbf{k})$. Namely, the real space coordinates do not commute with each other. With this commutator, the equation of motion for the wavepacket reads [9, 10]

$$
\begin{aligned}
\frac{d x_{\mu}}{d t} & =-i\left[x_{\mu}, H\right], \\
\frac{d \pi_{\mu}}{d t} & =-i\left[\pi_{\mu}, H\right] .
\end{aligned}
$$

Here the Hamiltonian $H$ is given by $H=\varepsilon_{n}(\mathbf{k})+V(\mathbf{x})$ with $\varepsilon_{n}(\mathbf{k})$ being the energy dispersion of the band $n$ of interest, and $V(\mathbf{x})$ is the slowly varying external potential. Putting this into eqs.(5) and we obtain

$$
\begin{aligned}
\frac{d x_{\mu}}{d t} & =\frac{\partial \varepsilon_{n}(\mathbf{k})}{\partial k_{\mu}}-i\left[x_{\mu}, x_{\nu}\right] \frac{\partial V(\mathbf{x})}{\partial x_{\nu}} \\
& =\frac{\partial \varepsilon_{n}(\mathbf{k})}{\partial k_{\mu}}+\varepsilon_{\mu \nu \lambda} b_{\lambda}(\mathbf{k}) \frac{\partial V(\mathbf{x})}{\partial x_{\nu}} \\
& =\frac{\partial \varepsilon_{n}(\mathbf{k})}{\partial k_{\mu}}+(\mathbf{b} \times \mathbf{F})_{\mu}, \\
\frac{d k_{\mu}}{d t} & =-\frac{\partial V(\mathbf{x})}{\partial x_{\mu}}=F_{\mu},
\end{aligned}
$$

where $\mathbf{F}=-\nabla_{\mathbf{x}} V(\mathbf{x})=e \mathbf{E}$ is the force acting on the electron. In eq.(6), the duality in the equation of motion is evident, i.e., the real space quantities $x_{\mu}, A_{\mu}(\mathbf{x})$, $V(\mathbf{x})$ and those in the momentum space $k_{\mu}, a_{n \mu}(\mathbf{k}), \varepsilon_{n}(\mathbf{k})$ enter into the equations of motion in a symmetric way. Therefore one can translate the phenomena in real (momentum) space to those in the momentum (real) space. Note, however, that compared with the real space magnetic field which is divergence-free, i.e., $\nabla_{\mathbf{x}} \cdot \mathbf{B}(x)=0$, corresponding to the absence of the magnetic monopole in real space, $\nabla_{\mathbf{k}} \cdot \mathbf{b}(\mathbf{k})$ can be non-zero in momentum space. Another important remark is that the symmetries give the following constraint. The time-reversal symmetry $T$ gives the relation $\mathbf{b}_{n}(\mathbf{k})=-\mathbf{b}_{n}(-\mathbf{k})$, while the inversion symmetry $I \mathbf{b}_{n}(\mathbf{k})=\mathbf{b}_{n}(-\mathbf{k})$. Therefore, when both $T$ and $I$ symmetries are there, there is no Berry curvature $\mathbf{b}_{n}(\mathbf{k})$. Also, in the noncentrosymmetric system where $I$-symmetry is absent, $\mathbf{b}_{n}(\mathbf{k})$ can be nonzero although the contributions from $\mathbf{k}$ and $-\mathbf{k}$ cancel with each other.

\section{B. Emergent SU(2) gauge field from Dirac equation}

Starting from the Dirac equation, the projection of the wavefunctions onto the low energy subspece leads to the gauge structure analogous to the electromagnetism. This formalism is based on the real-space picture, and is complementary to the discussion in the last subsection where the momentum space geometry has been considered. When the $\mathrm{SU}(2)$ spin space is preserved, it leads to the $\mathrm{SU}(2)$ non-Abelian gauge field which is coupled to the 
spin current, corresponding to the spin-orbit interaction (SOI).

We start with the quantum electrodynamics (QED), where the Dirac relativistic electrons and their charge current is minimally coupled to EMF. Therefore, it appears that there is no chance for the spin current to take some role in the electromagnetic phenomena. More explicitly, in the natural unit where $\hbar=c=1$, the Lagrangian of QED reads [ $[$ ]

$$
L=\bar{\psi}\left[i \gamma^{\mu} \hat{D}_{\mu}-m\right] \psi
$$

where $\psi$ is the 4-component spinor field, $\bar{\psi}=\psi^{\dagger} \gamma^{0}, \gamma^{\mu}$ is the Dirac matrices, and $\hat{D}_{\mu}=\partial_{\mu}-i e A_{\mu}$ is the gauge covariant derivative with $\mu=0,1,2,3$. The 4 -component charge current density is defined as

$$
j^{\mu}=-\frac{\partial L}{\partial A_{\mu}}=-e \bar{\psi} \gamma^{\mu} \psi
$$

whose 0-component is the charge density $\rho$, while the spatial components are the current density $\mathbf{j}$. From the gauge invariance, the conservation law of the charge is derived through Noether's theorem as

$$
\partial_{\mu} j^{\mu}=\frac{\partial \rho}{\partial t}+\nabla \cdot \mathbf{j}=0 .
$$

By taking the variation, one can derive the Maxwell equation

$$
\partial_{\mu} F^{\mu \nu}=j^{\nu}
$$

and Dirac equation

$$
\left[i \gamma^{\mu} \hat{D}_{\mu}-m\right] \psi=0
$$

As is well-known, the solutions to Dirac equation are classified into 2 classes, i.e., the positive energy and negative energy states separated by the twice of the rest mass energy of the electrons $2 m c^{2}$. Since the energy $m c^{2}$ is of the order of $\mathrm{MeV}$, for the low energy phenomena typically of the order of $\sim \mathrm{eV}$, the negative energy states are not relevant. Therefore, the non-relativistic Schroedinger equation is usually used which describes the dynamics of the 2-component spinor wavefunctions for positive energy states. However, one needs to take into account one important aspect of "projection". Namely, the negligence of the negative energy states means the projection of the wavefunctions onto the positive energy states, i.e, the sub-Hilbert space. Usually the sub-space is not flat but curved, and associated geometrical structure is introduced. The derivation of the effective Lagrangian describing the low energy physics is achieved by the expansion with respect to $1 /\left(m c^{2}\right)$, and the result reads [11 13

$L=i \psi^{\dagger} D_{0} \psi+\psi^{\dagger} \frac{\mathbf{D}^{2}}{2 m} \psi+\frac{1}{2 m} \psi^{\dagger}\left[e q \tau^{a} \mathbf{A} \cdot \mathbf{A}^{a}+\frac{q^{2}}{4} \mathbf{A}^{a} \cdot \mathbf{A}^{a}\right] \psi$, where $\psi$ is now the 2-component spinor and $D_{0}=$ $\partial_{0}+i e A_{0}+i q A_{0}^{a} \frac{\tau^{a}}{2}$, and $D_{i}=\partial_{i}-i e A_{i}-i q A_{i}^{a} \frac{\tau^{a}}{2}$ $(i=1,2,3)$ are the gauge covariant derivatives with $q$ being the quantity proportional to the Bohr magneton [11, 13]. $A_{\mu}$ is the vector potential for EMF, while the $\mathrm{SU}(2)$ gauge potential are defined as $A_{0}^{a}=B_{a}$, $A_{i}^{a}=\epsilon_{i a \ell} E_{\ell}$. The former is coupled to the charge current, and the latter to the 4-component spin current $j_{0}^{a}=\psi \sigma^{a} \psi, j_{i}^{a}=\frac{1}{2 m i}\left[\psi^{\dagger} \sigma^{a} D_{i} \psi-D_{i} \psi^{\dagger} \sigma^{a} \psi\right]$. Note that the spin current is the tensor quantity with one suffix for the direction of the spin polarization while the other for the direction of the flow. Note an important difference between the EMF and the SU(2) gauge field. The former has the gauge symmetry, i.e., the freedom to chose the arbitrary gauge for the vector potential $A_{\mu}$, while the "vector potential" $A_{\mu}^{a}$ for the latter is given by the physical field strength $\mathbf{B}$ and $\mathbf{E}$. Actually, the relation $\partial^{\mu} A_{\mu}^{a}=0$ holds. Therefore, the $\mathrm{SU}(2)$ gauge symmetry is absent. This is the basic reason why the spin is not conserved in the presence of the relativistic SOI. Instead, the spin current is "covariantly" conserved and satisfies $11-13$.

$$
D_{0} J_{0}^{a}+\mathbf{D} \cdot \mathbf{J}^{a}=0 .
$$

This means that in the co-moving frame the spin is conserved, while in the laboratory frame the spin source or sink appears when the electron forms a loop and comes back to the same position in space since the frame already changes [13]. (Note that the usual SU(2) gauge theory is a nonlinear theory and the gauge field is "charged", and the sum of the spin current by matter field and the gauge field is conserved in the non-Abelian gauge theory as Yang-Mills first showed [ [6]. However, the SU(2) gauge invariance is absent here, and also the action $\operatorname{Tr}\left[F_{\mu \nu}^{2}\right]$. There is no spin current from the non-Abelian gauge field when it is assumed to be the frozen background field.)

When the magnetic ordering occurs, the wavefunctions are further projected onto the spin component at each site, which is described by the field operator $\psi_{\sigma}$ of electrons decomposed into

$$
\psi_{\sigma}(\mathbf{x})=z_{\sigma}(\mathbf{x}) f(\mathbf{x})
$$

with $f$ being the spinless fermion corresponding to the charge degrees of freedom while $z_{\sigma}$ being the 2component spinor corresponding to the direction of the magnetization at $\mathrm{x}$. Putting eq.(14) into eq.(12), we obtain the effective Lagrangian for $f$-field as

$$
L_{\text {eff. }}=\psi^{\dagger}\left[i \partial_{0}+a_{0}^{B}+a_{0}^{S O}+A_{0}+\frac{\left(\nabla+i \mathbf{a}^{B}+i \mathbf{a}^{S O}+i e \mathbf{A}\right)^{2}}{2 m}\right] \psi,
$$

where $a_{\mu}^{B}=i\left\langle z\left|\partial_{\mu}\right| z\right\rangle$ is the $\mathrm{U}(1)$ field originating from the Berry connection of the spin wavefunctions, and $a_{\alpha}^{S O}=A_{\alpha}^{a}\left\langle z\left|\tau^{a} \partial_{\alpha}\right| z\right\rangle, a_{0}^{S O}=A_{0}^{a}\left\langle z\left|\tau^{a}\right| z\right\rangle$ are $\mathrm{U}(1)$ field originating from the SOI.

Here a remark about the geometrical meaning of the gauge field $a_{\mu}^{B}$ is in order [14]. The magnetic flux made from $\mathbf{a}^{B}$, i.e., $\mathbf{b}=\nabla \times \mathbf{a}^{B}$, corresponds to the solid angle 
subtended by the spins. Namely, the scalar spin chirality given by $\mathbf{S}_{i} \cdot\left(\mathbf{S}_{j} \times \mathbf{S}_{k}\right)$ is reduced to $\mathbf{b}$ along the direction normal to the plane made by the 3 sites $i, j$, $k$ in the continuum limit. Therefore, the effective magnetic field is produced when the spin structure is noncoplanar. In summary, there are three "electromagnetic fields" in magnetic systems, i.e., (i) $a^{B}$ due to the Berry phase associated with the non-coplanar spin structure, (ii) $a^{S O}$ from the spin-orbit interaction (SOI), and (iii) usual Maxwell EMF $A$.

\section{Emergent electromagnetic field in correlated electronic systems}

Up to now, we have considered the gauge fields in the band structure or magnetically ordered state, i.e., the single-particle properties, where the gauge fields are the static background fields. The electron correlation effect corresponds to the fluctuating spin beyond the mean field theory, and accordingly the gauge fields become dynamical [15]. This issue has been studied extensively in the research of high temperature superconductors [14, 16]. As the simplest example, let us consider the non-linear sigma model describing the low energy physics of the quantum antiferromagnet [4, 17].

$$
S=\frac{1}{g} \int_{0}^{\beta} d \tau \int d r\left|\partial_{\mu} \mathbf{n}\right|^{2},
$$

where the unit vector $\mathbf{n}$ specifies the staggered moment, and $g \propto 1 / S(S:$ spin quantum number $)$ is the dimensionless coupling constant representing the quantum fluctuation. We introduce the spinor field $z={ }^{t}\left(z_{\uparrow}, z_{\downarrow}\right)$ with the constraint $\sum_{\sigma} z_{\sigma}^{\dagger} z_{\sigma}=1, \mathbf{n}$ can be represented by $\mathbf{n}=z_{\sigma}^{\dagger}(\sigma)_{\sigma, \sigma^{\prime}} z_{\sigma^{\prime}}$ with $\sigma=\left(\sigma_{x}, \sigma_{y}, \sigma_{z}\right)$ are the Pauli matrices. Then $S$ in eq.(16) can be written as

$$
S=\frac{1}{g}\left|\left(\partial_{\mu}+i a_{\mu}\right) z_{\sigma}\right|^{2}
$$

with the gauge field $a_{\mu}=-i z_{\sigma}^{\dagger} \partial_{\mu} z_{\sigma}$ corresponds to the Berry phase connection between the 2 spinors at the neighboring 2 spatial points. Therefore, this $a_{\mu}$ corresponds to the $\mathrm{U}(1)$ gauge field due to the spin chirality discussed in the previous subsection. When we regard $z_{\sigma}$ as being the classical field, i.e., the condensed component corresponding to the magnetic ordering, the gauge field $a_{\mu}$ is reduced to $a^{B}$ discussed in the last subsection. Without the magnetic ordering, on the other hand, there is no condensation of $z$-field and hence the Higgs phenomenon for the gauge field $a_{\mu}$ does not occur. In this spin liquid state, this gauge field is the dynamical degrees of freedom, i.e., obtains the effective action by integrating over the rapid components of the spin fluctuations [15]. (Note that there is no term describing the dynamics of the gauge field at the starting since it is introduced to express the constraint.) Therefore the gauge field appears dynamically as emergent phenomenon. The quantum spin fluctuations and electron correlation effects are the subjects of intensive studies in condensedmatter physics, and the gauge theoretical approach has been a powerful tool [4, 5, 16]. Historically, motivated by the studies on strongly correlated systems and quantum Hall systems from the gauge theoretical viewpoint during 1990's, the conventional materials, which can be well described by band theory and/or the mean field theory, have been revisited from this new perspective. Below, we take some of the examples, where new physical effects have been explored in conventional systems from the viewpoint of gauge fields.

\section{MATERIALS AND PHENOMENA OF EMERGENT ELECTROMAGNETISM}

As described above, there are several sources of the EEMF, and correspondingly there are so many related phenomena in condensed-matters. The common feature of these phenomena is that the topological currents play an essential role. In solids, there are many imperfections such as the defects and impurities, which cause the scattering of electrons and dissipation. This dissipation, i.e., Joule heating, is balanced with the energy supplied from the external electric field. This is the usual Ohmic current. In the superconducting state, the macroscopic quantum coherence and the associated "rigidity" against the phase twist by the external magnetic field produces the superconducting current. This current does not decay nor cause any dissipation since the current-flowing state is in the thermal equilibrium, and is separated by the macroscopic energy barrier from the zero current state. To this list we would add here the third category of the current, i.e., the topological current, induced by the gauge field or curvature of the Hilbert space, i.e., EEMF. This third category includes the quantum Hall current and polarization current in ferroelectric materials, which are related to the Berry phase. This topological current does not require the off-diagonal long range order (ODLRO), and can exist in the normal states even at room temperature. Therefore, the topological currents will be of vital importance when the applications to electronics are considered. We will describe below several novel phenomena driven by this topological current in solids.

\section{A. Multiferroics}

The minimal coupling to the $\mathrm{SU}(2)$ gauge field $A_{\mu}^{a}$ in eq.(12) or in eq.(15) for magnets means the close relation between the spin current $j_{\mu}^{a}$ and the electric polarization $\mathbf{P}$. More explicitly, $\mathbf{P}$ is given by the derivative of the Lagrangian with respect to $\mathbf{E}$, i.e.,

$$
P_{i} \propto \epsilon_{i a \nu} j_{\nu}^{a}
$$


which means that the spin current produces the ferroelectric moment. Then the next problem is how one can produce the spin current. In the magnetically ordered state, the expectation value of the spin current $\left.\left\langle j_{\mu}^{a}\right\rangle\right\rangle$ in the ground state can be nonzero for the noncollinear spin configuration. Consider the expression of the spin current operator in the tight-binding model

$$
j_{\mu}^{a}=i \sum_{i} t_{i i+\mu} c_{i \alpha}^{\dagger}\left(\sigma^{a}\right)_{\alpha \beta} c_{i+\mu \beta}+h . c .,
$$

where $t_{i j}$ is the transfer integral, $\sigma^{a}(a=x, y, z)$ the Pauli matrices, and $\alpha, \beta$ the spin indices. For simplicity, let us consider the case of the spin ordering within the $x y$-plane, and the corresponding wavefunciton $\chi_{i}={ }^{t}\left(\frac{1}{\sqrt{2}}, \frac{e^{i \phi_{i}}}{\sqrt{2}}\right)$. Taking the expectation value with this wavefunction assuming the half-filling, one obtains

$$
\left\langle j_{\mu}^{z}\right\rangle \propto \frac{t_{i i+\mu}^{2}}{U} \sin \left(\phi_{i}-\phi_{i+\mu}\right)
$$

where we employed the perturbation theory in $t / U(U$ : on-site Coulomb interaction or the Hund's coupling). This is analogous to the Josephson current in superconductor with $\phi$ being the phase of the order parameter, and the spin supercurrent in magnet is induced by the tilted spins. Putting this into eq.(18), $\left\langle P_{z}\right\rangle \propto$ $\epsilon_{z \mu \nu} \sin \left(\phi_{i}-\phi_{i+\nu}\right)$. More general expression is easily obtained for the polarization $P_{i j}$ obtained by the 2 spins $\mathbf{S}_{i}$ and $\mathbf{S}_{j}$ as

$$
\mathbf{P}=\eta \mathbf{e}_{i j} \times\left(\mathbf{S}_{i} \times \mathbf{S}_{j}\right)
$$

with $\eta$ being the coupling constant related to SOI, and $\mathbf{e}_{i j}$ is the unit vector connecting the 2 sites $i$ and $j[18$, 19]. The vector product of the 2 spins $\chi_{i j}=\mathbf{S}_{i} \times \mathbf{S}_{j}$ is called vector spin chirality. This quantity is even with respect to the time-reversal operation $T$ similarly to the spin current.

This generic argument is applied to the real materials as follows. Compared with the free electrons in vacuum, the strength of the relativistic spin-orbit interaction can be enhanced by the factor of $\sim 10^{6}$ which is the ratio of the rest mass of the electrons $m c^{2}$ to the band gap. For $3 \mathrm{~d}$ electrons in transition metal atoms, the SOI $\lambda$ is typically of the order of $\sim 20-50 \mathrm{meV}$, while it becomes $\sim 0.5 \mathrm{eV}$ for $5 \mathrm{~d}$ electrons. The electron correlation energy, on the other hand, decreases from $3 \mathrm{~d}$ to $5 \mathrm{~d}$ since the wavefunction is more and more expanded. In the cubic crystal field in transition metal oxides, the 5-fold degeneracy of d-orbitals is lifted due to the ligand field of oxygens. As the result, 3 -fold degenerate $t_{2 g}$ orbitals ( $x y, y z, z x$-orbitals) with lower energy, and doubly degenerate $e_{g}$-orbitals $\left(x^{2}-y^{2}, 3 z^{2}-r^{2}\right.$-orbitals) with higher energy are formed. The matrix elements of the orbital angular momentum $\ell$ are zero within the $e_{g}$-orbitals. On the other hand, they are nonzero among $t_{2 g}$-orbitals and also between the $e_{g^{-}}$and $t_{2 g}$-orbitals. This SOI is the origin of the relativistic coupling between the magnetism and electric polarization. To give a more explicit prediction for transition metal oxides, the cluster model of magnetic ions sandwiching an oxygen ion has been studied theoretically by taking into account the spin-orbit interaction when deriving the super-exchange interaction [18]. As mentioned above, the spin current flows between the 2 non-collinear spins $\mathbf{S}_{i}$ and $\mathbf{S}_{j}$, which produces the electric polarization $\mathbf{P}$ as given by

$$
\mathbf{P} \cong-\frac{4 e}{9}\left(\frac{V}{\Delta}\right)^{3} I \mathbf{e}_{i j} \times\left(\mathbf{S}_{i} \times \mathbf{S}_{j}\right),
$$

where $I=\left\langle p_{x}|z| d_{z x}\right\rangle$, and $\Delta(V)$ is the energy difference (hybridization) between the p-orbitals and the d-orbitals. The SOI interaction is implicitly included in this model by picking up one doublet after splitting by the SOI. Applying this result to various magnetic structures, one can easily predict the presence or absence, and the direction of the polarization. This theory is not contradicting with the symmetry argument developed for magnets [20, 21], but stresses the physical mechanism of the spin currentinduced polarization.

A recent experimental breakthrough is the discovery of the multiferroic behavior in $R \mathrm{MnO}_{3}(R=\mathrm{Gd}, \mathrm{Tb}, \mathrm{Dy})$ [2, 3]. In these materials, the spontaneous electric polarization $P_{s}$ appears accompanied by the magnetic order, and they are necessarily strongly coupled. It has been also revealed that the magnetic structure which induces the electric polarization is the cycloidal spiral in good accordance with the theoretical prediction above [22 24]. Figure 4 shows the representative multiferroic behavior of $\mathrm{DyMnO}_{3}$. In this material, the spin rotation plane flop from bc- to ab-planes, and accordingly the direction of the electric polarization changes from c- to a-axes [3].

Now the extensive experimental studies have been done to explore the multiferroic materials, e.g., $\mathrm{Ni}_{3} \mathrm{~V}_{2} \mathrm{O}_{8}$ [25], $\mathrm{Ba}_{0.5} \mathrm{Sr}_{1.5} \mathrm{Zn}_{2} \mathrm{Fe}_{12} \mathrm{O}_{22}$ [26], $\mathrm{CoCr}_{2} \mathrm{O}_{4}$ [27], $\mathrm{MnWO}_{4}$ [28], $\mathrm{CuFeO}_{2}[29], \mathrm{LiCuVO}_{4}[30]$, and $\mathrm{LiCu}_{2} \mathrm{O}_{2}$ [31] are discovered to be multiferroics. Namely, multiferroicity is not a special phenomenon but is a rather ubiquitous phenomenon in Mott insulators. These experimental findings urged the systematic theoretical studies on the microscopic mechanisms of the spin-related electric polarization [32, 33]. The perturbative approach in both $V / \Delta$ and $\lambda / \Delta$ is employed, where $V$ and $\Delta$ represent the transfer integral and the charge transfer energy between the transition-metal (TM) $d$ and ligand (L) $p$ orbitals. This analysis concludes that the polarization $\mathbf{P}_{\mathbf{r}+\frac{\mathrm{e}}{2}}$ appearing on the bond between the sites $\mathbf{r}$ and $\mathbf{r}+\mathbf{e}$ is given by

$$
\begin{aligned}
\mathbf{P}_{\mathbf{r}+\frac{\mathbf{e}}{2}} & =P^{\mathrm{ms}}\left(\mathbf{m}_{\mathbf{r}} \cdot \mathbf{m}_{\mathbf{r}+\mathbf{e}}\right) \mathbf{e}+\mathbf{P}^{\mathrm{sp}} \mathbf{e} \times\left(\mathbf{m}_{\mathbf{r}} \times \mathbf{m}_{\mathbf{r}+\mathbf{e}}\right) \\
& +P^{\mathrm{orb}}\left[\left(\mathbf{e} \cdot \mathbf{m}_{\mathbf{r}}\right) \mathbf{m}_{\mathbf{r}}-\left(\mathbf{e} \cdot \mathbf{m}_{\mathbf{r}+\mathbf{e}}\right) \mathbf{m}_{\mathbf{r}+\mathbf{e}}\right],
\end{aligned}
$$

where $\mathbf{m}_{\mathbf{r}}$ is the spin direction at $\mathbf{r}$. The first term $P^{\mathrm{ms}} \propto(V / \Delta)^{3}$ is the polarization due to the exchangestriction, which is nonzero when the inversion symmetry between $\mathbf{r}$ and $\mathbf{r}+\mathbf{e}$ is absent because the 2 intermediate states of doubly occupied d-orbitals becomes inequivalent. This term does not requires the SOI, hence is considered to be larger than the rest of the terms if it exits. 
The second term $P^{\mathrm{sp}} \propto(\lambda / \Delta)(V / \Delta)^{3}$ is due to the spin current mechanism already discussed. The third term $P^{\text {orb }} \sim \min (\lambda / V, 1)(V / \Delta)$ is nonzero for the partially filled $t_{2 g}$ orbitals and comes from the modification of the single-spin anisotropy due to the electric field [32, 33]. These three contributions appear differently depending on the wavevector of the polarization. Now the origins of the multiferroic behavior of various materials are investigated and are classified according to these 3 mechanisms in most of the cases [3] .

In eq. (23), the polarization is due to the relativistic SOI (spin current mechanism or spin anisotropy mechanism) or the broken inversion symmetry at the center of the bond (exchange-striction). Therefore, it is an important question what is the most ubiquitous and generic interaction between the electric field and the spin system without these mechanisms. By a careful analysis of the super-exchange processes in Mott insulators taking into account the quantum dynamics of the spins in the intermediate states, we have derived the following effective Lagrangian [34]

$$
L_{E}=-\int d^{3} \mathbf{r} T_{a b} E_{a} e_{b},
$$

where $\mathbf{E}$ is the electric field and

$$
\mathbf{e}=\frac{1}{2} \sin \theta\left(\partial_{t} \theta \nabla \varphi-\partial_{t} \varphi \nabla \theta\right)
$$

is the electric field component of EEMF associated with the spin structure derived from the U(1) Berry connection. Here $\mathbf{n}=(\cos \varphi \sin \theta, \sin \varphi \sin \theta, \cos \theta)$ is the unit vector of the magnetization in the continuum approximation, and $T_{a b}$ is the tensor which depends on the details of the atomic configurations. One important remark here is that $T_{a b}$ vanishes in the single-orbital case since the spins form singlet and has no quantum dynamics in the intermediate states in the processes of the super-exchange interaction. However, in most of the cases, the orbital degrees of freedom is active and $T_{a b}$ is nonzero. This coupling is analogous to the spin motive force in the metallic ferromagnetic systems by which the domain wall or vortex motions produce the voltage drop [35 37].

\section{B. Topological Hall effects}

As explained in eq.(6), the most natural phenomena expected from the gauge fields in momentum space are related to the transverse motion of the electrons to the external electric field, i.e., the Hall effects. Contrary to the usual Hall effect driven by the Lorentz force, we call the Hall effects originating from the gauge fields as topological Hall effects, and we describe below some of the examples.

\section{Spin-orbit interaction and anomalous Hall effect}

Hall discovered the Hall effect in metallic ferromagnetic systems due to the spontaneous magnetization instead of the external magnetic field 38]. This effect is called anomalous Hall effect (AHE) and its mechanism has been a controversial issue for more than a century. It is agreed that the effect is due to the SOI combined with the spontaneous magnetization, but the issue was the role of the impurity scatterings. Intrinsic mechanism was first proposed by Karplus-Luttinger (KL) 39], who considered the anomalous velocity for the first time discussed in section I. However, their theory has been criticized by Smit [40] saying that the impurity scattering, which is inevitable and also indispensable to reach the steady state under electric field, invalidates the KL theory. This posed the question if the dissipationless topological current can survive even in the presence of the dissipative Ohmic current. Smit [40] proposed the skew scattering mechanism, where the impurity scattering in the presence of SOI gives the asymmetry of the transition rates between $W_{\mathbf{k} \rightarrow \mathbf{k}^{\prime}}$ and $W_{\mathbf{k}^{\prime} \rightarrow \mathbf{k}}$. This breakdown of the detailed balance leads to the net current perpendicular to the applied electric field, i.e., the Ohmic transport current is slightly distorted in the perpendicular direction. Later Berger [41] proposed another mechanism called side-jump, where the transverse shift of the electron trajectory occurs at the scattering in the presence of the SOI. These 2 mechanisms are called extrinsic ones as opposed to the intrinsic one by KL. The recent advances in this problem is two-folds [38]. One is the recognition that the band structures of the ferromagnetic materials can be topologically nontrivial characterized by Chern numbers. Haldane [42] was the first to notice that the quantum Hall effect can be realized without the Landau level formation in a tight-binding model on a lattice with the complex transfer integrals. What is recognized recently is that this scenario can be realized in the ferromagnetic materials with the SOI [43]. In this case, even though the overlaps of the band dispersions make the system metallic, one can define the Chern number of each band when the gap opens at each $\mathbf{k}$-point. Therefore, the ferromagnetic metal could be an implicit quantum Hall system with $k_{z}$-dependent Chern numbers. The other is the state-of-art first-principles calculations of electronic states and the Hall response as well as the accurate experimental measurements of the AHE in various materials [38]. In short, the transverse conductivity $\sigma_{x y}$ due to the intrinsic mechanism can be written in terms of the Berry phase curvature, i.e., the magnetic flux $b_{n z}(\mathbf{k})$ for the Bloch wave state with crystal momentum $\mathbf{k}$ and the band index $n$ [44]:

$$
\sigma_{x y}=\frac{e^{2}}{2 \pi \hbar} \sum_{n \mathbf{k}} f\left(\varepsilon_{n}(\mathbf{k})\right) b_{n z}(\mathbf{k}),
$$

where the $f\left(\varepsilon_{n}(\mathbf{k})\right)$ is the Fermi distribution function for the energy $\varepsilon_{n}(\mathbf{k})$ of the Bloch state, and the vector poten- 
tial $a_{n \mu}(\mathbf{k})$ is defined in eq.(2), and $\mathbf{b}_{n}(\mathbf{k})=\nabla_{k} \times \mathbf{a}_{n}(\mathbf{k})$. The meaning of this expression is that the Hall current is the sum of the anomalous velocities of Bloch states occupied by electrons in the equilibrium distribution. Therefore the transverse conductivity $\sigma_{x y}$ represents the gauge field distribution in the momentum space. Especially when the chemical potential is in the gap, the $\mathbf{k}$-integral in eq. (26) is over the whole first Brillouin zone (1st BZ), and one might think it is zero due to the periodicity with respect to $\mathbf{k} \rightarrow \mathbf{k}+\mathbf{G}$ with $\mathbf{G}$ being the reciprocal lattice vector. Namely the contour integral over boundary of the 1st BZ appears to cancel. However this is not always the case, and the integral is $\left(e^{2} / h\right) \times$ integer especially in the 2-dimensions. This integer is the topological number called Chern number for the $U(1)$ fiber bundle of the Bloch wavefunction [44]. The finite Chen number means that the phase of the Bloch wavefunction can not be defined continuously over the whole 1st BZ analogous to the Yang-Wu construction of Dirac monopole [4, [5, 45]. Here the plural patches need to be introduced to cover the 1st BZ. The relevance of the Dirac monopole to the Hall effect is understood more explicitly in the following 3-dimensional model.

$$
H(\mathbf{k})=\sum_{a=x, y, z} k_{a} \sigma_{a} .
$$

The corresponding magnetic field associated with the Berry phase reads

$$
\mathbf{b}_{ \pm}(\mathbf{k})= \pm \frac{\mathbf{k}}{2|\mathbf{k}|^{3}}
$$

where \pm corresponds to the 2 bands with energy $\varepsilon_{ \pm}(\mathbf{k})=$ $\pm|\mathbf{k}|$. This means that the magnetic monopole exists at the band crossing point $\mathbf{k}=\mathbf{0}$, and $\nabla_{k} \cdot b_{ \pm}(\mathbf{k})= \pm 2 \pi \delta(\mathbf{k})$ has the delta-functional singularity. The contribution from the fixed $k_{z}$ is given by

$$
\sigma_{x y}\left(k_{z}\right)= \pm \frac{e^{2}}{2 h} \operatorname{sign} k_{z} .
$$

For the 2-dimensional system where $k_{z}$ is regarded as a parameter $m$ characterizing the system, it describes the quantum Hall system with $\sigma_{x y}= \pm \frac{e^{2}}{2 h}$. The factor $1 / 2$ is not realized in real system because the Dirac fermion appears always in the pair, i.e., species doubling, in the 1st BZ and hence the Chern number is an integer.

Essential to AHE is the fact that the nontrivial topological structure described above is not special as in the case of quantum Hall system, but is generic for magnets in the presence of the SOI. A simple 2-dimensional 3 -band model for $t_{2 g}$ orbitals was constructed, and the uniform magnetization produces the nonzero Chern number for each band [43]. In the absence of the SOI, the up and down spin bands are independent of each other except the exchange energy splitting, and there occurs several band crossing points but no finite Chern numbers. Then SOI lifts the degeneracies to generate the mass term $m$ as discussed above and produce the finite
Chern numbers. This is the reason why the SOI can not be treated perturbatively in sharp contrast to the previous theoretical treatments. This nontrivial behavior has been found both in the first-principles band calculation and in the experiment in $\mathrm{SrRuO}_{3}$, a metallic ferromagnet with $T_{c}=130 \mathrm{~K}$ [46]. In Figs. 5 shown the transport properties of $\mathrm{SrRuO}_{3}$. Figure 5(c) shows the temperature dependence of the Hall resistivity which is dominated by the anomalous contribution. Just below $T_{c}$, it is negative and turns into positive and again has the maximum. This characteristic behavior strongly suggests that the perturbative expansion in $\lambda M$ ( $\lambda$ : SOI, $M$ : magnetization) is not allowed and the AHE is a fingerprint of the Berry phase of the Bloch wavefunction. We plot in Fig.5(d) the transverse conductivity $\sigma_{x y}$ as a function of the spontaneous magnetization compared with the first-principles band structure calculation. This non-monotonous temperature dependence including the sign change is due to the band crossings acting as the magnetic monopoles in momentum space. Accordingly Fig. 6 shows the distribution of the Berry curvature $b_{n z}(\mathbf{k})$ as a function of $\left(k_{x}, k_{y}\right)$ at fixed $k_{z}=0$. It shows a sharp peak around $k_{x}=k_{y}=0$ and the ridges along $k_{x}= \pm k_{y}$. This sharp peak represents the monopole corresponding to the band crossing. When the Fermi energy $\varepsilon_{F}$ is near the magnetic monopoles, the electrons are subject to the strong gauge field and hence the contribution to the Hall constant is resonantly enhanced. The integral over $k_{z}$ leads to the partial cancellation of the positive and negative contributions, but still the rapid change of $\sigma_{x y}$ as a function of $\varepsilon_{F}$ results. This explains both the first-principle band calculation and experimental results, which are shown in Figs.5(c) and (d). Similar conclusion is obtained also for the AHE in Fe [47], where the sharp spiky structures of the Berry phase distribution has been found.

An important recent development is the experimental measurements of dynamical AHE, i.e., $\sigma_{x y}(\omega)$ in ferromagnetic materials. Thanks to the rapid progress of the telahertz spectroscopy [48, 49] , the low frequency structures of the dynamical AHE are being revealed. Especially, the dominance of the contributions from the band crossings is expected to appear in the $\omega$-dependence of $\sigma_{x y}(\omega)$ as well as the non-monotonous temperature dependence of $\sigma_{x y}(\omega=0)$. Figure 7 shows an example of the low frequency data for $\mathrm{AHE}$ in $\mathrm{SrRuO}_{3}$. It is clearly seen that nontrivial structure exists at around $4 \mathrm{meV}$, which is well fitted by a model assuming the band crossing near the Fermi energy.

Up to now, it is argued that in some materials, the AHE can be explained by the intrinsic mechanism based on the Berry phase concept, but the controversy between the intrinsic and extrinsic needs to be resolved. As for this problem, again the role of the enhanced Berry curvature near the band crossings and the topological nature are crucial. In ref. [50] a model with a band anticrossing is considered and its Hall response is calculated taking into account the disorder scatterings. The Hall conductivity $\sigma_{x y}$ is obtained as a function of $\sigma_{x x}$ repre- 
senting the strength of the disorder. Figure 8 summarizes the results including the various experimental data plotted. (Note that the calculation has been done for a $2 \mathrm{D}$ model, and $\sigma$ 's are scaled by the lattice constant along the $z$-direction which is assumed to be $\cong 0.4 \mathrm{~nm}$.) This result captures the respective role of the transport and topological currents. In the very clean region where $\sigma_{x x}>10^{6} \Omega^{-1} \mathrm{~cm}^{-1}$, the skew scattering mechanism (extrinsic mechanism) is dominant and hence $\sigma_{x y} \propto \sigma_{x x}$. When the disorder strength becomes larger, the transport current is suppressed much more rapidly compared with the topological current, the latter of which is "protected" topologically, leading to the plateau region in the region $10^{4} \Omega^{-1} \mathrm{~cm}^{-1}<\sigma_{x x}<10^{6} \Omega^{-1} \mathrm{~cm}^{-1}$. In this intermediate region, to which many of the metallic ferromagnets belong, the intrinsic mechanism is dominant and hence the first-principles calculations can predict the AHE semiquantitatively. When the disorder becomes even stronger, $\sigma_{x y}$ begins to decrease obeying the approximate scaling law $\sigma_{x y} \propto \sigma_{x x}^{1.6}$. This scaling is in good accordance with the experimental results as seen in Fig. 8 , but its understanding is still left for future studies. To conclude this subsection, AHE offers an interesting laboratory where both the Ohmic transport and topological currents coexist, and it has been established that the latter can contribute to the Hall effect even in the metallic systems. Quantized version of the AHE, where most of the bulk states are localized [51] or the chemical potential is in the gap [52], have been discussed also. In this case, the current is carried by the edge channels as in the case of quantum Hall system.

\section{Scalar spin chirality and anomalous Hall effect}

There are a vast variety of the non-collinear spin structures found experimentally. These systems are the ideal laboratory to test the idea of U(1) EEMF discussed in section I. Of particular interest is the idea of "scalar spin chirality" $\chi_{i j k}$ defined for the 3 spins as

$$
\chi_{i j k}=\mathbf{S}_{i} \cdot\left(\mathbf{S}_{j} \times \mathbf{S}_{k}\right),
$$

which is T-odd. As discussed in section IB and Fig.2, the scalar spin chirality acts as an effective magnetic field and leads to the Hall response [53, 54]. First, we consider the case where the magnetic unit cell is small, and the Bloch wavefunctions are defined in the 1st BZ. The total gauge flux penetrating the unit cell is zero or integer multiple of $2 \pi$ due to the periodicity since the contour integral of $\mathbf{a}(r)$ along its boundary vanishes $(\bmod 2 \pi)$ due to the periodicity. On the other hand, the gauge field corresponding to the Berry phase becomes nonzero for the Bloch wavefunctions in the momentum space with a possible finite Chern number of each band. Roughly speaking, this happens when the unit cell contains the more than 2 different types of the loop, and each band feels the fluxes with different weight to obtain the Chern number. Ohgushi et al. [54] showed that this idea can be realized in a ferromagnet on Kagome lattice with the non-coplanar spin configuration.

Pyrochlore lattice can be regarded as the 3 -dimensional generalization of the Kagome lattice, i.e., pyrochlore lattice contains the Kagome lattice normal to the [111] direction. In the material $\mathrm{Nd}_{2} \mathrm{Mo}_{2} \mathrm{O}_{7}$ (hereafter we denote it as $\mathrm{NMO}$ ), there are 2 interpenetrating sublattices composed of the tetrahedrons of $\mathrm{Nd}$ and Mo atoms shifted along the c-axis as shown in Fig.9(a) 55]. The conduction electrons are on the Mo sublattice, while the localized spins on Nd sublattice are subject to the strong anisotropy to form non-coplanar spin configurations. The localized spins and conduction electron spins are coupled antiferromagnetically, which transmit the scalar spin chirality of $\mathrm{Nd}$ to conduction electrons (Fig.9(b)). Figure 9 (c) shows the temperature dependence of the Hall resistivity which shows the steep increase as the temperature is lowered corresponding to the increase of the exchange field from $\mathrm{Nd}$ spins and associated scalar spin chirality. The absolute value of the low temperature value is consistent with the theoretical calculation taking into account the tilting angle of the Mo spins estimated from the neutron scattering experiment [55]. Furthermore, the observation of the sign change of $\sigma_{x y}$ under the external magnetic field along [111] direction [56] is consistent with the sign change of the spin chirality.

The analysis in $\mathbf{k}$-space above is justified when the magnetic unit cell is small compared with the mean free path $\ell$. In this case, the EEMF is defined by the Berry phase in $\mathbf{k}$-space. In the other limit, i.e., when $\ell$ is shorter than the size of the spin texture $\xi$, the EEMF is defined more appropriately in $\mathbf{r}$-space, which acts as the effective EMF with $\omega_{c} \tau<<1\left(\omega_{c}\right.$ : cyclotron frequency, $\tau$ : mean free time). This situation is realized in the recently discovered Skyrmion crystals in noncentrosymmetric magnets with $\mathrm{B} 20$ structure [57, 58]. $\mathrm{MnSi},(\mathrm{Fe}, \mathrm{Co}) \mathrm{Si}$, and MnGe are the examples of this class of materials, which has Dzyaloshinskii-Moriya (DM) interaction as described by the following Hamiltonian in the continuum approximation.

$H=\int d \mathbf{r}\left[\frac{J}{2}(\nabla \mathbf{M}(\mathbf{r}))^{2}+\alpha \mathbf{M}(\mathbf{r}) \cdot(\nabla \times \mathbf{M}(\mathbf{r}))-\mathbf{H} \cdot \mathbf{M}(\mathbf{r})\right]$,

where $\mathbf{M}(\mathbf{r})$ is the magnetization, $\mathbf{H}$ the magnetic field, $J$ the ferromagnetic interaction, and $\alpha$ the DM interaction constant. The lattice constant is taken to be unity. The ground state under zero magnetic field is the single spiral with the spins rotating in the plane perpendicular to the wavevector $\mathbf{q}$. The length of $q=|\mathbf{q}|$ is determined to the the ratio $\alpha / J$ while its direction is determined by the weak spin anisotropies not included in the Hamiltonian eq.(31).

Recently, a neutron scattering experiment identified the mysterious A-phase in $\mathrm{MnSi}$ as the Skyrmion crystal state stabilized by the external magnetic field and thermal fluctuations [57]. The conical spin structure is the most stable state under the magnetic field in the major part of the phase diagram in $3 \mathrm{D}$ crystal. On the 
other hand, when one reduces the thickness of the sample smaller than the wavelength of the spiral, the conical state becomes energetically higher than the Skyrmion crystal when the external magnetic field is perpendicular to the film. Actually, a Monte Carlo simulation of the 2D magnet with DM interaction concluded that the Skyrmion crystal state is stable in much wider region of the phase diagram in $(T, B)$-plane ( $T$ : temperature, $B$ : magnetic field ) including the zero temperature case 59 . Motivated by this study, a recent experiment using the Lorentz tunneling electron microscopy (TEM) succeeded in real-space observation of the Skyrmion crystal in the thin film of $(\mathrm{Fe}, \mathrm{Co}) \mathrm{Si}$ 58]. Figure 10 shows the phase diagram and the Lorentz TEM images of the Skyrmions and Skyrmion crystals. The left panel shows the experimental results, while the right panel the results by Monte Carlo simulations, which indicate the good agreement.

As shown in Fig. 11 schematically, each Skyrmion wrap the sphere once in the order parameter space of $\mathbf{M}$, which means that the integral of the magnetic flux $\mathbf{b} \| \hat{z}$ over one Skyrmion is $2 \pi$. From this one can estimate the effective magnetic field induced by the Skyrmion. It is typically $4000 \mathrm{~T}$ when the size of the Skyrmion $\xi=1 \mathrm{~nm}$, and inversely proportional to the square of $\xi^{2}$. Since $\mathrm{MnSi}$, (Fe,Co)Si, MnGe, are metallic systems, the conduction electrons are coupled to the spins and spin chirality. Therefore, the Hall effect is expected due to the Lorentz force driven by $\mathbf{b}$ replacing the external magnetic field B. Recently, the Hall measurement on MnGe has been done and the contribution from the Skyrmion has been analyzed [60]. The Hall resistivity $\Delta \rho_{y x} \cong 200 \mathrm{n} \Omega \mathrm{cm}$ is due to this topological contribution, which is corresponding to the effective magnetic field $b \cong 1100 T$ for $\xi=3 \mathrm{~nm}$. These values are compared with the case of $\mathrm{MnSi}$, where $\xi=18 \mathrm{~nm}, b \cong 28 T$, and $\Delta \rho_{y x} \cong 5 \mathrm{n} \Omega \mathrm{cm}$.

The dynamics of Skyrmions and Slyrmion crystal is an intriguing issue to be studied. A recent experiment [61] shows the Skyrmion crystal motion driven by a current with the threshold value $j_{c} \cong 10^{2} \mathrm{~A} / \mathrm{cm}^{2} \mathrm{~m}$, which is orders of magnitude smaller than that for the domain wall motion in ferromagnets 62]. Theoretically, the motion of the Skyrmions will induce the electric field e as electromotive force due to $d \mathbf{b} / d t$. This leads to several physical consequences such as a new mechanism for the damping of the Skyrmion motion, and the motion of the Skyrmion transverse to the current (Skyrmion Hall effect) 63].

\section{Hall effect of light}

The topological Hall effect does not require that the particles are charged, and hence is possible even for the uncharged particles. A representative example of uncharged particle is the photon, and one can ask if the Hall effect of light can occur or not. The constraint which induces the Berry curvature in this case is that the electric and magnetic fields of light is always perpendicular to the direction of the propagation, i.e., that of the wavevector $\mathbf{k}$. This situation corresponds to the strong coupling limit of SOI in electrons. Actually, the effect of the Berry phase in the propagation of light in the optical fiber has been studied both experimentally and theoretically, and the rotation of the polarization was confirmed for the light with the $\mathbf{k}$-vector slowly changing and enclosing an area on the sphere [64].

Note that the quantum nature of the photon is not required for this Berry phase effect, which is solely due to the wave nature of light. Therefore, this offers a unique opportunity to study the Berry phase effect in classical systems. Extending this idea, we have derived the semiclassical equation of motion for the wavepacket of light including the effect of the finite wavelength 65). (Note that the wave-optics is reduced to the geometric optics in the short-wavelength limit, the former of which corresponds to the quantum mechanics while the latter to the classical Newtonian mechanics.) This semiclassical equations of motion for the position $\mathbf{r}$, the wavevector (momentum) $\mathbf{k}$ and the spin state $|z\rangle$ (2-component spinor describing the polarization of light) reads

$$
\begin{aligned}
\frac{d \mathbf{r}}{d t} & =v(\mathbf{r}) \frac{\mathbf{k}}{|\mathbf{k}|}+\frac{d \mathbf{k}}{d t} \times\left\langle z\left|\boldsymbol{\Omega}_{\mathbf{k}}\right| z\right\rangle, \\
\frac{d \mathbf{k}}{d t} & =-[\nabla v(\mathbf{r})] \mathbf{k}, \\
\frac{d|z\rangle}{d t} & =-i \frac{d \mathbf{k}}{d t} \cdot \boldsymbol{\Lambda}_{\mathbf{k}}|\mathbf{z}\rangle,
\end{aligned}
$$

where $v(\mathbf{r})=c / n(\mathbf{r})$ is the velocity of light in the medium, $\left[\boldsymbol{\Lambda}_{\mathbf{k}}\right]_{\lambda \lambda^{\prime}}=-i \mathbf{e}_{\lambda \mathbf{k}}^{\dagger} \nabla_{\mathbf{k}} \mathbf{e}_{\lambda^{\prime} \mathbf{k}}$ is the connection of $2 \times 2$ matrix form, i.e., $\mathrm{SU}(2)$ gauge connection, and $\boldsymbol{\Omega}_{\mathrm{k}}=\nabla_{\mathrm{k}} \times \boldsymbol{\Lambda}_{\mathrm{k}}+\boldsymbol{\Lambda}_{\mathrm{k}} \times \boldsymbol{\Lambda}_{\mathrm{k}}$ is the field strength. It is diagonal in the basis of the right and left-circular polarization, i.e., $\boldsymbol{\Omega}_{\mathbf{k}}=\sigma_{3} \mathbf{k} /|k|^{3}$. This equation indicates that the Berry curvature has the opposite sign for opposite circularly polarized light, which gives the polarizationdependent anomalous velocity adding to the group velocity of wavepacket. More explicitly, one can consider the reflection and transmission of light at the interface of 2 media with different dielectric constants [65]. The anomalous velocity is finite when the particle is subject to the gradient of the refractive index $\nabla n(\mathbf{r})$, and results in the finite transverse shifts of the transmitted and reflected lights at this reflection/transmission. These shits are in the opposite direction for opposite circular polarization. This effect has recently been observed experimentally using the "weak measurement" 66.

The idea of the Berry phase for light can be generalized to the photonic crystal, where the Bloch waves are formed. Especially, one can consider the crystal with distortion, where the Berry phase is defined in the 6dimensional space $(\mathbf{k}, \mathbf{r})$. Especially, the curvature is enhanced near the gap edge when the gap is small. This situation is realized for the X-ray propagating in crystals since the deviation of the dielectric function from unity is typically of the order of $10^{-6}$, and hence the "periodic 
potential" for the Bloch wave is very weak. Sawada et al. 67] considered the role of this enhanced Berry curvature in the propagation of X-ray in deformed crystal. The prediction is that the shift in the trajectory $\Delta \mathbf{r}$ of the $\mathrm{X}$-ray is given by

$$
\Delta \mathbf{r} \cong \mathbf{G}(\mathbf{G} \cdot \mathbf{u}) \frac{\omega}{\Delta \omega} \frac{1}{|\mathbf{k}|^{2}}
$$

where $\mathbf{G}$ is the reciprocal lattice vector satisfying the Bragg condition for the wavevector $\mathbf{k}$ of the X-ray, $\mathbf{u}$ the displacement of the crystal, $\omega$ the frequency of $\mathrm{X}$ ray, and $\Delta \omega$ is the gap in the Bloch wave of X-ray. As mentioned above, $\omega / \Delta \omega$ can be as large as $10^{6}$, which determines the magnification of $\Delta \mathbf{r}$ compared with $\mathbf{u}$ considering that $|\mathbf{G}| \sim|\mathbf{k}|$. This theoretical prediction has been recently confirmed experimentally using the single crystal of Si [68], and offers a new principle for the X-ray microscope.

\section{Hall effect of magnons}

Another example of the uncharged particle is the magnons in insulating magnets. Although the charge current is not available in this case, the energy current can be carried by the magnons and hence the thermal Hall effect without resorting to the Lorentz force is When one considers the propagation of the magnon wave in a ferromagnet, it behaves as a Bloch wave and the band structure is formed. When more than 2 atoms are in the unit cell, the Berry phase is generally expected for this Bloch wavefunction. We consider the Hamiltonian with the Dzyaloshinskii-Moriya (DM) SOI as

$$
H=-\sum_{i j} J_{i j} \mathbf{S}_{i} \cdot \mathbf{S}_{j}+\mathbf{D}_{i j} \cdot\left(\mathbf{S}_{i} \times \mathbf{S}_{j}\right)-\sum_{i} \mathbf{H} \cdot \mathbf{S}_{i}
$$

Suppose the external magnetic field $\mathbf{H}$ and ferromagnetic moments align along $+z$-direction. Then, the spin wave Hamiltonian can be written as

$$
H_{S W}=-\sum_{i j} \tilde{J}_{i j} S\left(e^{-i \phi_{i j}} b_{i}^{\dagger} b_{j}+\text { h.c. }\right)+\sum_{i} H^{z} b_{i}^{\dagger} b_{i}
$$

where $\tilde{J}_{i j} e^{i \phi_{i j}}=J_{i j}+i D_{i j}^{z}$. This indicates that the DM interaction acts as a vector potential and effective magnetic flux for the propagating magnons [69]. Similar to the case of AHE, the distribution is such that the total effective magnetic flux is zero (or equivalently the integer multiple of $2 \pi$ ) when integrated over the unit cell. Therefore, finite effect survives when the inequivalent loops exist in the unit cell. Kagome lattice and its $3 \mathrm{D}$ generalization pyrochlore lattice are the representative crystal structures satisfying this condition. And actually the thermal Hall effect has been recently observed in an insulating ferromagnet $\mathrm{Lu}_{2} \mathrm{~V}_{2} \mathrm{O}_{7}$ with pyrochlore structure [69]. In the pyrochlore structure, the midpoint between any 2 corners of a tetrahedron is not an inversion symmetry center, and hence the nonzero DM interaction is expected. Symmetry further determines the direction of the DM vectors $\mathbf{D}_{i j}$ as shown in Fig. 12(a).

In this material, the spontaneous magnetization $M$ emerges below Curie temperature $T_{c}=70 \mathrm{~K}$, which is isotropic and almost coincides with 1 Bohr magneton $\left(\mu_{B}\right)$ at low temperatures, indicating the collinear ferromagnetic state with spin $S=1 / 2$. Below $T_{c}$, the thermal Hall conductivity $\kappa_{H}$ is discernible, whereas it is very small above $80 \mathrm{~K}$. The magnitude of the thermal Hall conductivity has a maximum at around $50 \mathrm{~K}$. Similar to the magnetization, the thermal Hall conductivity steeply increases and saturates in the low magnetic field region. Therefore, the observed thermal Hall effect is due to the spontaneous magnetization $M$ as in the case of AHE. $\kappa_{H}$ gradually decreases with increasing the magnetic field furthermore due to the opening of the gap in the magnon spectrum induced by the magnetic field. All those effects are contained in following theoretical expression for $\kappa_{\alpha \beta}$ [69]:

$$
\begin{aligned}
\kappa_{\alpha \beta} & =\Phi_{\alpha \beta} \frac{k_{B}^{2} T}{p i^{3 / 2} \hbar a}\left(2+\frac{g \mu_{B} H}{2 J S}\right)^{2} \\
& \times \sqrt{\frac{k_{B} T}{2 J S}} \operatorname{Li}_{5 / 2}\left[\exp \left(-\frac{g \mu_{B} H}{k_{B} T}\right)\right],
\end{aligned}
$$

where $\Phi_{\alpha \beta}=-\varepsilon_{\alpha \beta \gamma} n_{\gamma} D /(8 \sqrt{2} J)$ ( $\varepsilon_{\alpha \beta \gamma}$ : totally antisymmetric tensor, $\mathbf{n}$ : direction of the magnetization, $a$ : lattice constant, $\left.D=\left|\mathbf{D}_{i j}\right|\right)$, and $\operatorname{Li}_{n}(z)=$ $\sum_{k=1, \infty} z^{k} / k^{n}$. Figure 12(b) shows the comparison between the experimental result and eq.36). The only adjustable parameter is the ratio $D / J=0.32$, which is a reasonable value for transition metal oxides. From these facts, it is convincing that the thermal Hall effect observed in $\mathrm{Lu}_{2} \mathrm{~V}_{2} \mathrm{O}_{7}$ is due to the magnons affected by the Berry curvature due to the DM interaction.

\section{Spin Hall effect}

The concept of the anomalous velocity can be generalized to the time-reversal $T$-symmetric systems. As already discussed in eq. (32), the SU(2) Berry curvature is non-zero even in the $T$-invariant system, and the analogous effect is expected for the electrons also. This idea has been explored for the band structure of semiconductors [70]. In GaAs and $\mathrm{Ge}$, the valence bands consist of three p-ortbitals $(L=1)$ while the conduction bands s-orbitals $(L=0)$. Therefore the 6 bands (3 times the spin degeneracy 2 ) constitute the valence bands, which are split into 2-fold degenerate split-off (SO) bands $(J=1 / 2)$, and the 4 -fold degenerate bands $(J=3 / 2)$ at the $\Gamma$-point $(k=0)$. The 4 -fold degenerate bands are further split into 2 doubly-degenerate heavy-hole and light-hole bands, i.e., 2 Kramers degenerate bands at finite $\mathbf{k}$. (Here we neglect the breaking of the inversion symmetry in the case of GaAs, which is small.) 
These 4 bands are described by Luttinger Hamiltonian as

$$
\begin{aligned}
H_{0} & =\sum_{\mathbf{k}} c_{\mu, \mathbf{k}}^{\dagger} H_{\mu \nu}(\mathbf{k}) c_{\nu, \mathbf{k}} \\
H_{\mu \nu}(\mathbf{k}) & =\frac{1}{2 m}\left(\left(\gamma_{1}+\frac{5}{2} \gamma_{2}\right) k^{2}-2 \gamma_{2}(\hat{\mathbf{k}} \cdot \mathbf{S})^{2}\right)_{\mu \nu}
\end{aligned}
$$

As discussed above, the degeneracy acts as the magnetic monopole for the Berry curvature, and the $\Gamma$-point is especially interesting since the 2 doubly-degenerate bands touch there. Since there are Kramers degeneracy at each $\mathbf{k}$-point, the Berry connection is the $2 \times 2$ matrix $(S U(2))$. There are 2 pseudo-spin states, i.e., parallel and anti-parallel pseudo-spin to $\hat{\mathbf{k}}=\mathbf{k} /|\mathbf{k}|$, which is called helicity. The gauge field and hence the anomalous velocity depend on the helicity of the state, i.e., it has the opposite sign for different helicity states. Therefore, even though there is no net charge current, the external electric field can induce the transverse spin current. This is called the (intrinsic) spin Hall effect, and for the case of the p-doped GaAs/Ge the spin current $j_{\alpha}^{\mu}$ was predicted to be 70 ]

$$
J_{j}^{i}=\sigma_{H}^{s} \epsilon^{i j k} E_{k}
$$

with

$$
\sigma_{H}^{s}=\frac{1}{6 \pi^{2}}\left(k_{F}^{H}-k_{F}^{L}\right)
$$

where $k_{F}^{H}\left(k_{F}^{L}\right)$ is the Fermi wavenumber for the heavy (light) holes. This intrinsic spin Hall effect is driven by the band structure and its Berry phase connection, in sharp contrast to the extrinsic mechanisms previously proposed by Dyakonov and Perel in 1971 [71] and others [72, 73]. Sinova et al. independently proposed the intrinsic spin Hall effect for n-GaAs using the Rashba model 74]. Note that eq.(38) looks natural when one remember that the $\mathrm{SU}(2)$ gauge potential $A_{i}^{a}=\epsilon_{\text {ia }} E_{\ell}$ is coupled to the spin current as discussed in section I. However, the topological structure of the Bloch wavefunction is needed to realize the intrinsic spin Hall effect.

There are several experimental reports on the spin Hall effect. In semiconductors, the spin accumulation near the edge of the sample is measured to confirm the spin Hall effect. Kato et al. [75] observed the spin accumulation at the edge of the n-type GaAs in terms of the Kerr rotation. Wunderlich et al. [76] observed the circular polarized LED from the recombination process of the holes in the p-type GaAs. Both experiments seems to be relevant to the spin Hall effect, but its origin, i.e., intrinsic or extrinsic, is still controversial [77]. The experiment on metals usually measures the inverse spin Hall effect, i.e., the voltage induced by the injection of spin current from the ferromagnetic metals [78]. For some of the metals, the first-principles band structure calculations are applied to predict the intrinsic spin Hall effect in e.g. Pt, which agrees reasonably well with the experimental observations [79]. The readers are referred to a review [7] for more details.

\section{OTHER INTERESTING SYSTEMS AND CONCLUSIONS}

There are several important and intriguing systems from the viewpoint of EEMF which are not covered in section II. One natural question to ask is "What is the global aspect of the sub-Hilbert space and gauge fields, and their implications on the physical properties ?". Of particular importance from this viewpoint is the topological insulators [80]. One can imagine the 2 copies of quantum Hall systems with opposite chiralities for up and down spins. This systems will show the quantized spin Hall conductance due to the nonzero Chern numbers $C_{\uparrow}=-C_{\downarrow}$. The spin Chern number is defined as $C_{\uparrow}-C_{\downarrow}$, which can be finite even for the $T$-symmetric systems. However, in the presence of the SOI, usually all the components of the spin are not conserved, and hence the spin current and Chern number are not well defined. Actually, the spin Hall conductance in spin Hall insulator, where the chemical potential is inside the band gap but still the spin Hall conductance is finite due to the band inversion, is not quantized [82? ]. Kane-Mele discovered a $T$-symmetric model with SOI, which shows a stable nontrivial topological phase with the helical edge channels even with the mixing of the spins [80]. This phase is characterized by the $Z_{2}$ topological number instead of the Chern number. This idea is now generalized to 3-dimensions and also to arbitrary dimensions to classify all the possible topologically nontrivial states as far as the band structure can be defined [83]. The basic concept is that the Bloch wavefucntions as the fiber bundle can have the globally nontrivial structure characterized by the index defined as the integral of the Berry curvature or EEMF over the 1st BZ. Combining the mapping between the different dimensions and symmetry class enables the classification of the topological classes including both the insulators and superconductors with gap. Recently the generalization to include the $\mathbf{k}$ - and $\mathbf{r}$-spaces has been achieved also [84]. The readers are referred to recent reviews for more details 80 .

Noncentrosymmetric systems with SOI are also interesting laboratory to study the topological nature and EEMF. Rashba system is the representative one described by the Hamiltonian

$$
H_{R}=\frac{\mathbf{p}^{2}}{2 m}+\lambda \mathbf{e}_{z} \cdot(\mathbf{s} \times \mathbf{p}),
$$

where $\mathbf{e}_{z}$ is the direction of the potential gradient which breaks $I$-symmetry, $\mathbf{s}$ and $\mathbf{p}$ are the spin and momentum operators, respectively. This interaction has been experimentally demonstrated for the 2-dimensional electrons at the interface of GaAs system [85] or at the interface electrons in oxides system [86, 87], and also the 
bulk 3-dimensional material BiTeI [88]. The spin splitting at each $\mathbf{k}$-point means that the spin and the velocity is tightly coupled, and hence the transport and magnetic properties. Therefore, the various cross correlations between the magnetic and transport degrees of freedom are expected and actually observed experimentally in the Rashba systems, which constitute the important part of the spintronics. The spin Hall effect, spin Galvanic effect, and dynamical magneto-electric effect are the examples of this cross-correlation driven by the Rashba interaction [89, 90]. Combined with the superconducting order parameter, the Rashba type SOI leads to even more interesting phenomena [91]. Because of the absence of the inversion symmetry, the classification of even and odd parity does not work there, and hence the singlet and triplet pairings are mixed. This fact leads to several unusual features such as the $H_{c 2}$ beyond the Pauli limit [91]. Furthermore, it is shown that the topological superconductor with helical Majorana edge channels as the Andreev bound states can be realized when the p-wave pairing is dominant over the s-wave pairing component [92]. When the magnetic field or exchange field is applied to open the gap at the band crossing and the Fermi energy is within that gap, the spinless pairing superconductor is realized, and the Majorana fermions are expected to appear there 93]. These are consistent with the more general classification scheme of the topological superconductors [83].

In this article, we have discussed the physics of emergent electromagnetic field (EEMF) in condensed-matter systems. The gauge connections and fields are naturally introduced in various situations when the wavefunctions are constrained on some manifold in the Hilbert space. In the band structure and/or in the mean field theory, this gauge fields are not dynamical but frozen one or are controlled as parameters by the external electromagnetic field (EEM). However, the physical phenomena driven by EEMF are even richer than those of EMF because the lattice gauge theory, non-Abelian gauge fields, higher dimensions, topological terms, are often relevant to EEMF. In this sense, the gauge fields can be a guiding and unifying principle in condensed-matter physics. Needless to say, the dynamical nature of EEMF in correlated systems and spin liquids, which was not covered in this article, also remains to be an important subject, and continue to be a central issue in the condensed-matter theory [4, 5, 17].

The author thanks H. Katsura, M. Mochizuki, S. Murakami, N. Furukawa, A.V.Balatsky, M. Onoda, S. Onoda, H.J.Han, C. Jia, K. Nomura, M.Mostovoy, S.C. Zhang for collaborations, and T. Arima, N. Kida, M. Kawasaki, D. I. Khomskii, and A. Aharony for useful discussions. This work was supported by Priority Area Grants, Grant-in-Aids under the Grant numbers 19048015, 19048008, and 21244053, and NAREGI Nanoscience Project from the Ministry of Education, Culture, Sports, Science, and Technology, Japan, Strategic International Cooperative Program (Joint Research Type) from Japan Science and Technology Agency, and by Funding Program for World-Leading Innovative R and D on Science and Technology (FIRST Program)D
[1] P. Curie, J. Phys. 3, 393 (1894).

[2] T. Kimura et al., Nature 426, 55 (2003).

[3] For recent reviews, Y. Tokura, Science 312, 1481 (2006); S.-W. Cheong and M. Mostovoy, Nat. Mater. 6, 13 (2007); Y. Tokura, J. Magn. Magn. Mater. 310, 1145 (2007); M. Fiebig, J. Phys. D: Appl. Phys. 38, R123 (2005); Y. Tokura, Science 312, 1481 (2006).

[4] E. Fradkin, Field Theories of Condensed Matter Systems, (Addison Wesley, 1991).

[5] A. Altland and B. Simons, Condensed Matter Field Theory, (Cambridge Univ. Press, 2006).

[6] See for example M.E. Peshkin and D.V. Schroeder, Introduction to Quantum Field Theory (Addison-Wesley, New York, 1995).

[7] A. Shapere and F. Wilczek: Geometric Phases in Physics (World Scientific, Singapore, 1989).

[8] M. V. Berry: Proc. Roy. Soc. London A 392 (1984) 45.

[9] G. Sundaram and Q. Niu: Phys. Rev. B 59 (1999) 14915.

[10] E. N. Adams and E. I. Blount: J. Phys. Chem. Solids 10 (1959) 286.

[11] J. Froelich and U.M. Studer, Rev. Mod. Phys. 65, 733 (1993).

[12] G. Volovik, The Universe in a Helium Droplet (Oxford University Press, USA,2003).

[13] B.W.A. Leurs, Z. Nazario, D.I. Santiago, and J. Zaanen, Annals of Physics 323, 907 (2008).
[14] See for example Patrick A. Lee and Naoto Nagaosa. Phys. Rev. B 46, 5621 (1992).

[15] See for example A.M. Polyakov, Gauge fields and Strings, (harwood academic publishers, 1987).

[16] Patrick A. Lee, Naoto Nagaosa, and Xiao-Gang Wen, Rev. Mod. Phys. 78, 17 (2006).

[17] Xiao-Gang Wen, Quantum Field Theory of Many-Body Systems, ( Oxford Univ. Press, 2004).

[18] H. Katsura, N. Nagaosa, and A. V. Balatsky, Phys. Rev. Lett. 95, 057205 (2005).

[19] N. Nagaosa, J. Phys. Cond.-Mat. 20, 434207 (2008); N. Nagaosa, J. Phys. Soc. Jpn. 77, 031010 (2008).

[20] L.D. Landau, E.M. Lifshitz and L.P. Pitaevskii, Electrodynamics of Continuous Media ( Elsevier, Oxford, 2008).

[21] A.B. Harris and G. Lawes, in The Handbook of Magnetism and Advanced Magnetic Materials, ed. H. Kronmuller and S. Parkin, (Wiley, 2006); A.B. Harris et al., Phys. Rev. B 73, 184433 (2006).

[22] M. Kenzelmann et al., Phys. Rev. Lett.95, 087206 (2005).

[23] Y. Yamasaki, H. Sagayama, T. Goto, M. Matsuura, K. Hirota, T. Arima, and Y. Tokura, Phys. Rev. Lett. 98, 147204 (2007)

[24] Y. Yamasaki et al., Phys. Rev. Lett. 101, 097204 (2008).

[25] G. Lawes, A. B. Harris, T. Kimura, N. Rogado, R. J. Cava, A. Aharony, O. Entin-Wohlman, T. Yildirim, M. Kenzelmann, C. Broholm, and A. P. Ramirez, Phys. Rev. 
Lett. 95, 087205 (2005).

[26] T. Kimura, G. Lawes, and A. P. Ramirez, Phys. Rev. Lett. 94, 137201 (2005).

[27] Y. Yamasaki, S. Miyasaka, Y. Kaneko, J.-P. He, T. Arima, and Y. Tokura, Phys. Rev. Lett. 96, 207204 (2006).

[28] K. Taniguchi, N. Abe, T. Takenobu, Y. Iwasa, and T. Arima, Phys. Rev. Lett. 97, 097203 (2006).

[29] T. Kimura, J. C. Lashley, and A. P. Ramirez, Phys. Rev. B 73, 220401 (2006).

[30] Y. Naito, Kenji Sato, Yukio Yasui, Yusuke Kobayashi, Yoshiaki Kobayashi, and Masatoshi Sato, condmat/0611659.

[31] S. Park, Y. J. Choi, C. L. Zhang, and S-W. Cheong, Phys. Rev. Lett. 98, 057601 (2007).

[32] C. Jia, S. Onoda, N. Nagaosa, and J.H. Han., Phys. Rev. B 74, 224444 (2006).

[33] C. Jia, S. Onoda, N. Nagaosa, and J. H. Han, Phys. Rev. B 76, 144424 (2007).

[34] M. Mostovoy, K. Nomura, and N. Nagaosa, arXiv:1010.3687.

[35] L. Berger, Phys. Rev. B 33, 1572 (1986).

[36] S.E. Barnes and S. Maekawa, Phys. Rev. Lett. 98, 246601 (2007).

[37] S.A. Yang et al., Phys. Rev. Lett. 102, 067201 (2009).

[38] N. Nagaosa, J. Sinova, S. Onoda, A. H. MacDonald, and N. P. Ong, Rev. Mod. Phys. 82, 1539 (2010).

[39] R. Karplus and J. M. Luttinger, Phys. Rev. 95, 1154 (1954).

[40] J. Smit, Physica Amsterdam 21, 877 (1955): ibid 24, 39 (1958).

[41] L. Berger, Phys. Rev. B 2, 4559 (1970).

[42] F.D.M. Haldane, Phys. Rev. Lett. 61, 2015 (1988).

[43] M. Onoda, N. Nagaosa, J. Phys. Soc. Jpn. 71, 19 (2002).

[44] D. J. Thouless, M. Kohmoto, M. P. Nightingale and M. den Nijs: Phys. Rev. Lett. 49, 405 (1982); M. Kohmoto: Ann. Phys. (N.Y.) 160, 343 (1985).

[45] P. A. M. Dirac: Proc. Roy. Soc. London 133, 60 (1931).

[46] Z. Fang, N. Nagaosa, K. S. Takahashi, A. Asamitsu, R. Mathieu, T. Ogasawara, H. Yamada, M. Kawasaki, Y. Tokura and K. Terakura: Science 30292 (2003).

[47] Yugui Yao, Leonard Kleinman, A. H. MacDonald, Jairo Sinova, T. Jungwirth, Ding-sheng Wang, Enge Wang and Qian Niu: Phys. Rev. Lett. 92037204 (2004).

[48] S. Iguchi, S. Kumakura, Y. Onose, S. Bordacs, I. Kezsmarki, N. Nagaosa, and Y. Tokura Phys. Rev. Lett. 103, 267206 (2009)

[49] R. Shimano, Y. Ikebe, K. S. Takahashi, M. Kawasaki, N. Nagaosa and Y. Tokura1, Europhys. Lett. 95, 17002 (2011).

[50] S. Onoda, N. Sugimoto, and N. Nagaosa, Phys. Rev. Lett. 97, 126602 (2006); Phys. Rev. B 77, 165103 (2008).

[51] Masaru Onoda and Naoto Nagaosa, Phys. Rev. Lett. 90, 206601 (2003).

[52] R. Yu, W. Zhang, H.J. Zhang, S.C. Zhang, X. Dai, and Z. Fang, Science 329, 61 (2010).

[53] J. Ye, Y. B. Kim, A. J. Millis, B. I. Shraiman, P. Majumdar and Z. Tesanovic: Phys. Rev. Lett. 83 (1999) 3737 .

[54] K. Ohgushi, S. Murakami and N. Nagaosa: Phys. Rev. B 62 (2000) R6065.

[55] Y. Taguchi, Y. Oohara, H. Yoshizawa, N. Nagaosa and Y. Tokura: Science 291 (2001) 2573.

[56] Y. Taguchi, T. Sasaki, S. Awaji, Y. Iwasa, T. Tayama,
T. Sakakibara, S. Iguchi, T. Ito and Y. Tokura: Phys. Rev. Lett. 90 (2003) 257202.

[57] S. Muhlbauer et al., Science 323, 915 (2009).

[58] X.Z. Yu et al., Nature 465, 901 (2010).

[59] S.D. Yi, S. Onoda, N. Nagaosa, and J.H. Han, Phys. Rev. B 80, 054416 (2009).

[60] N. Kanazawa et al., Phys. Rev. Lett. 106, 156603 (2011).

[61] F. Janietz et al., Science 330, 1648 (2010).

[62] A. Yamaguchi, T. Ono, S. Nasu, K. Miyake, K. Mibu, and T. Shinjo, Phys. Rev. Lett. 92, 077205 (2004).

[63] Jiadong Zang, Maxim Mostovoy, Jung Hoon Han, Naoto Nagaosa, arXiv:1102.5384.

[64] R. Y. Chiao and Y. S. Wu, Phys. Rev. Lett. 57, 933 (1986); A. Tomita and R. Y. Chiao, ibid. 57, 937 (1986); M. V. Berry, Nature 326, 277 (1987).

[65] Masaru Onoda, Shuichi Murakami, Naoto Nagaosa, Phys. Rev. Lett. 93, 083901 (2004).

[66] O. Hosten and P. Kwiat, Science 319, 787 (2008).

[67] Kei Sawada and Naoto Nagaosa, Phys. Rev. Lett. 95, 237402 (2005).

[68] Yoshiki Kohmura, Kei Sawada, and Tetsuya Ishikawa, Phys. Rev. Lett. 104, 244801 (2010).

[69] Y. Onose et al., Science 329(5989), 297 (2010).

[70] S. Murakami, N. Nagaosa, and S.-C. Zhang: Science 301 (2003) 1348.

[71] M. I. Dyakonov and V. I. Perel: JETP Lett. 13 (1971) 467.

[72] J. E. Hirsch: Phys. Rev. Lett. 83 (1999) 1834.

[73] S. Zhang: Phys. Rev. Lett. 85 (2000) 393.

[74] J. Sinova, D. Culcer, Q. Niu, N. A. Sinitsyn, T. Jungwirth and A. H. MacDonald: Phys. Rev. Lett. 92 (2004) 126603.

[75] Y. K. Kato, R. C. Myers, A. C. Gossard and D. D. Awschalom: Science 306 (2004) 1910.

[76] J. Wunderlich, B. Kaestner, J. Sinova and T. Jungwirth: Phys. Rev. Lett. 94 (2005) 047204.

[77] S. Murakami and N. Nagaosa, Vol.1 in Comprehensive Semiconductor Science and Technology (SEST) Edited by Pallab Bhattacharya, Roberto Fornari, and Hiroshi Kamimura. (Elsevier, 2011).

[78] S. O. Valenzuela, M. Tinkham, Nature 442, 176 (2006); E. Saitoh, M. Ueda, H. Miyajima, and G. Tatara, Appl. Phys. Lett. 88, 182509 (2006); T. Kimura et al., Phys. Rev. Lett. 98156601 (2007).

[79] G. Y. Guo, S. Murakami, T.-W. Chen, and N. Nagaosa, Phys. Rev. Lett. 100, 096401 (2008).

[80] M. Z. Hasan and C. L. Kane, Rev. Mod. Phys. 82, 3045 (2010); X.-L. Qi and S.-C. Zhang: arXiv:1008.2026.

[81] Note that a early work has discussed the spin quantum Hall effect was discussed in superfluid $\mathrm{He}^{3}$. G.E. Volovik, AIP Conference Proceedings 194 136-146 (1989).

[82] S. Murakami, N. Nagaosa, and S.-C. Zhang: Phys. Rev. Lett. 93, 156804 (2004).

[83] A. Schnyder, S. Ryu, A. Furusaki, and A.W.W. Ludwig, Phys. Rev. B 78, 195125 (2008).

[84] Jeffrey C. Y. Teo and C. L. Kane, Phys. Rev. B 82, 115120 (2010).

[85] J. Nitta, T. Akazaki, H. Takayanagi, and T. Enoki, Phys. Rev. Lett. 78, 1335 (1997).

[86] A. Ohtomo and H. Y. Hwang, Nature 427, 423 (2004).

[87] N. Reyren et al., Science 317, 1196 (2007).

[88] Ishizaka, et. al., Nature Materials 10, 521 (2011).

[89] A. Chernyshov et al., Nature Phys. 5, 656 (2009).

[90] I.M. Miron et al., Nature Materials 9, 230 (2010). 
[91] E. Bauer, et. al., Phys. Rev. Lett. 92, 027003 (2004); P. A. Frigeri, et. al., Phys. Rev. Lett. 92, 097001 (2004).

[92] Yukio Tanaka, Takehito Yokoyama, Alexander V. Balatsky, and Naoto Nagaosa, Phys. Rev. B 79, 060505 (2009).

[93] Yukio Tanaka, Masatoshi Sato, and Naoto Nagaosa, arXiv:1105.4700. 


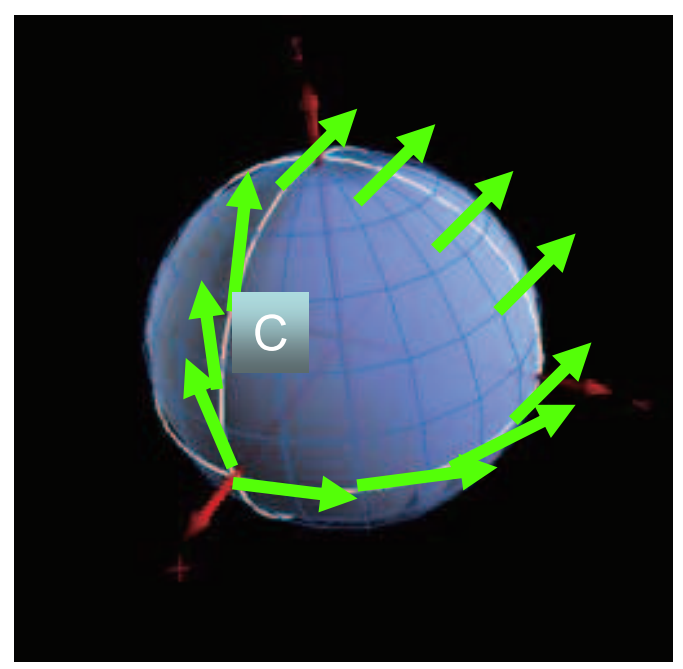

Fig.1 Parallel transport of a vector on the sphere. Going around a loop, the direction of the vector has been changed corresponding to the solid angle enclosed by the loop. 


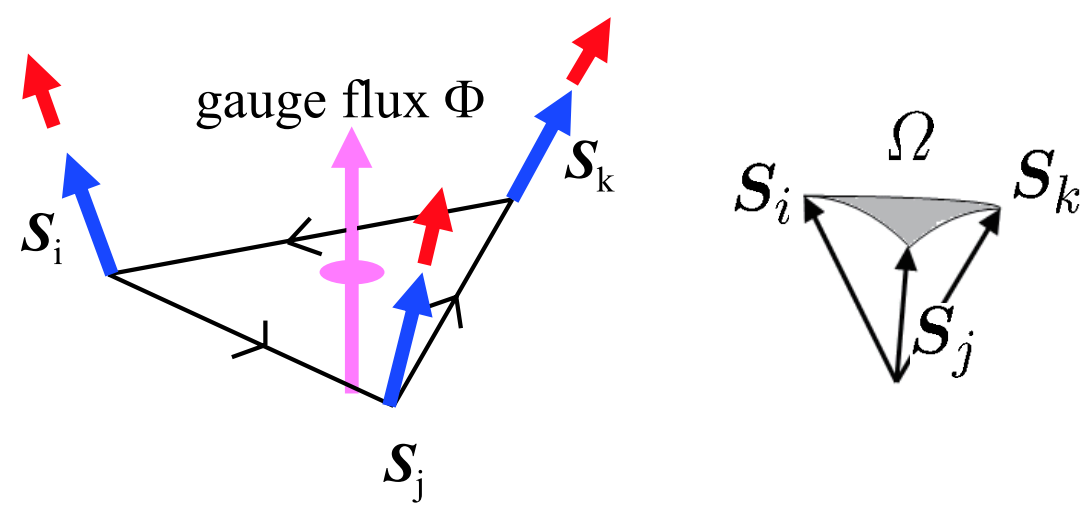

Fig.2 Scalar spin chirality as the gauge field for the conduction. When the spin directions S's form the solid angle $\Omega$, the conduction electrons whose spins (red arrows) are coupled to S's feel $\Omega / 2$ as the gauge flux. 


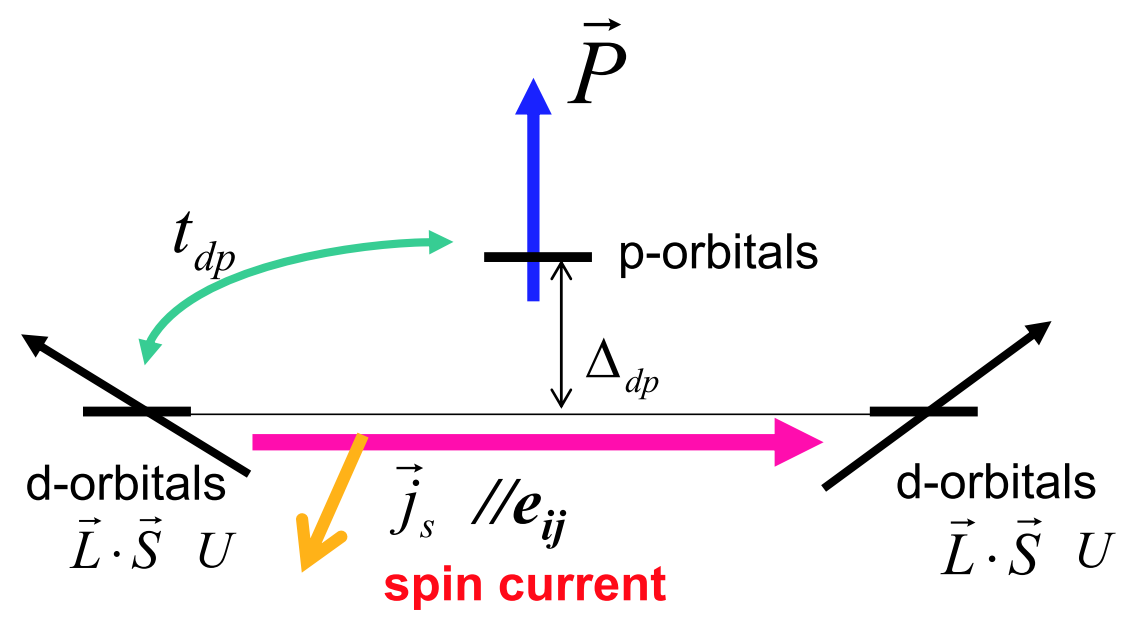

Fig.3 Cluster model for the spin current mechanism of the electric polarization. The red arrow represent the direction of the flow and the orange arrow that of the Spin polarization of the spin current, which produces the electric polarization $P$ through the spin-orbit interaction on the d-orbitals. 


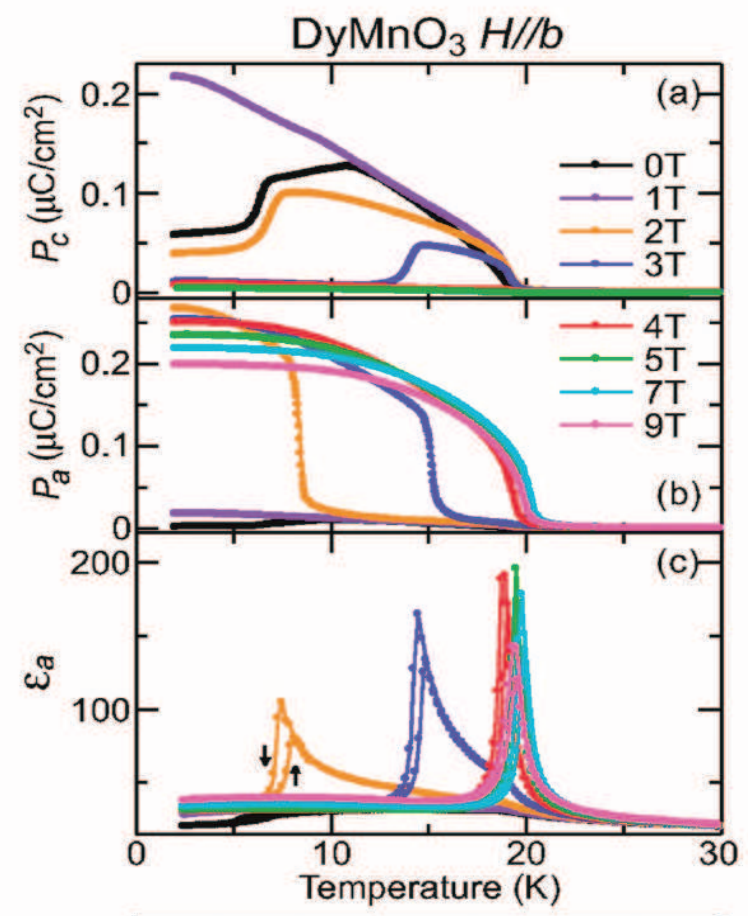

Fig.4 Experimental observation of the multiferroic behavior of DyMnO3. (a) and (b) show the temperature dependence of the spontaneous electric polarization along $c$ and a axes, respectively, for several values of magnetic field. There occurs the flop of The polarization under the magnetic field. (c)and (d) show the corresponding temperature dependence of the dielectric constant along a-axis.

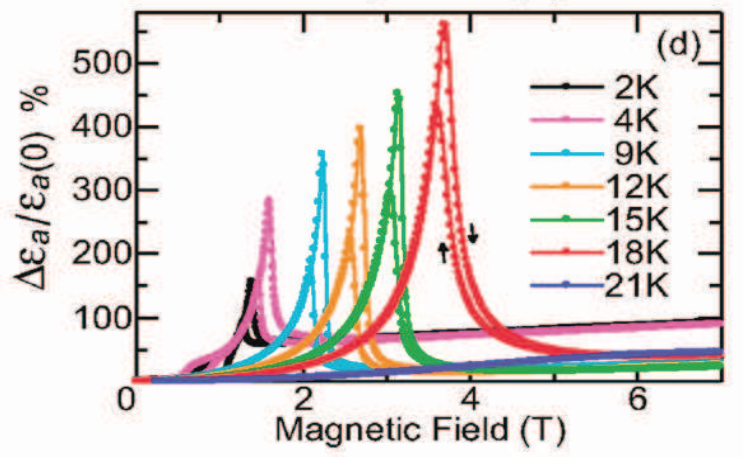



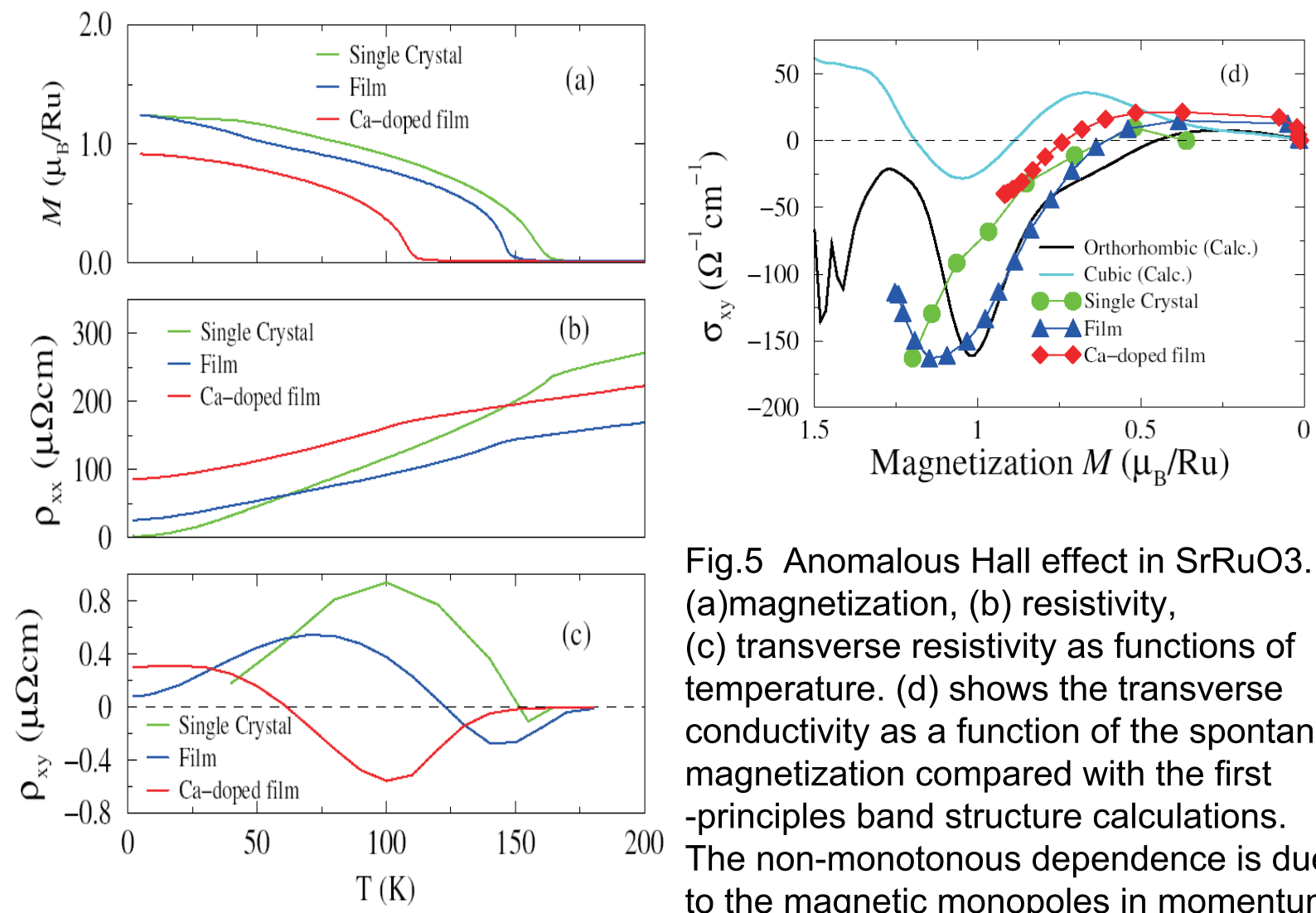

Fig.5 Anomalous Hall effect in SrRuO3. (a)magnetization, (b) resistivity, (c) transverse resistivity as functions of temperature. (d) shows the transverse conductivity as a function of the spontaneous magnetization compared with the first -principles band structure calculations. The non-monotonous dependence is due to the magnetic monopoles in momentum space. 


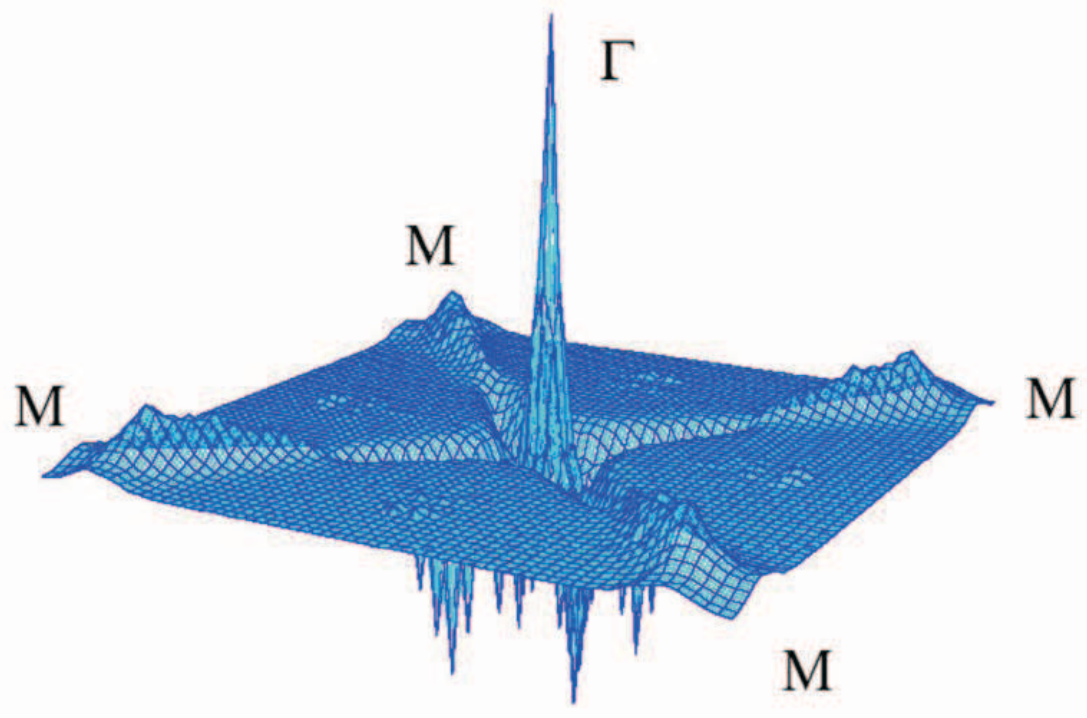

Fig.6 Distribution of the Berry curvature in momentum space as a function of $(\mathrm{kx}, \mathrm{ky})$ at $\mathrm{kz}=0$ calculated for SrRuO3. The sharp peak at $\Gamma$-point is due to the band (anti)crossing acting as the magnetic monopole. 


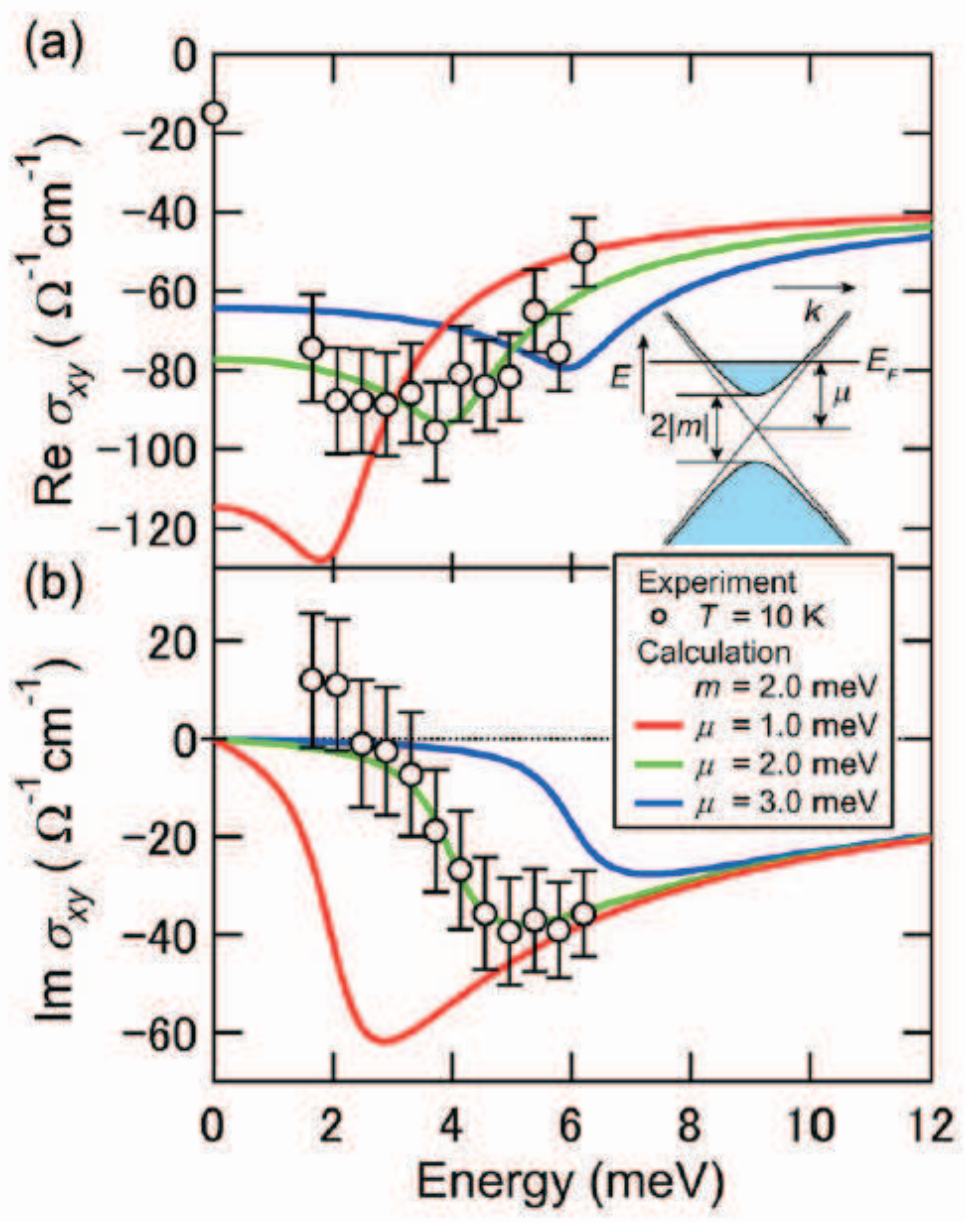

Fig.7 Finite frequency anomalous Hall conductivity of SrRuO3 in the Telahertz region. The observed behavior is well fitted by the simple model of the anti-crossing near the Fermi energy. 


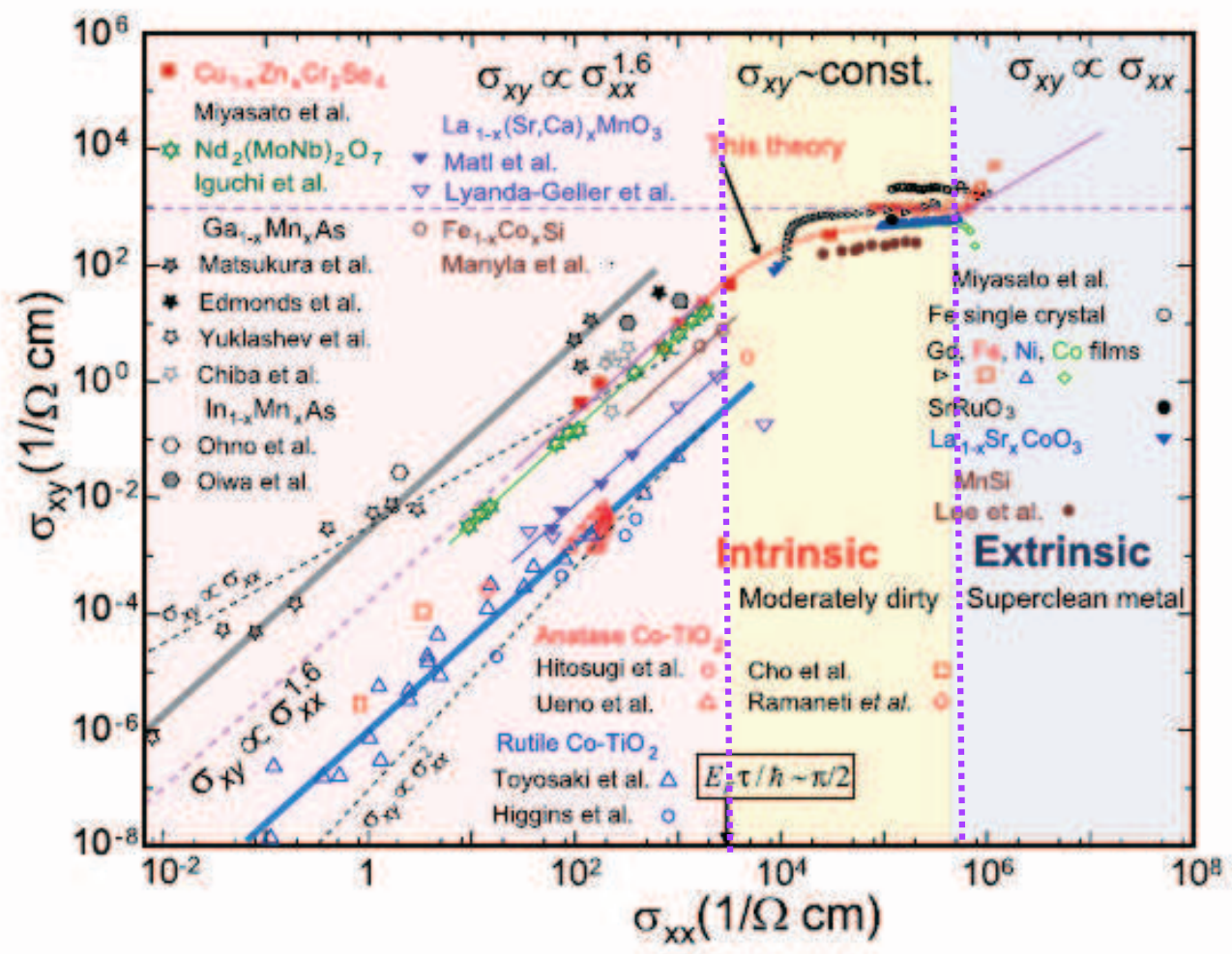

Fig.8 Anomalous Hall conductivity as a function of the longitudinal conductivity calculated for a two-dimensional model with the anti-crossing near the Fermi energy ( pink curve). Various experimental data are also plotted. 


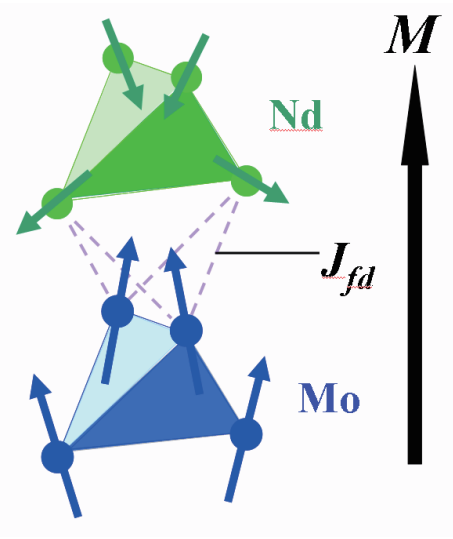

(a)

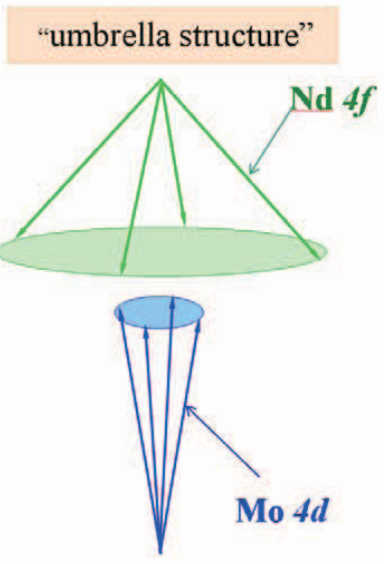

(b)

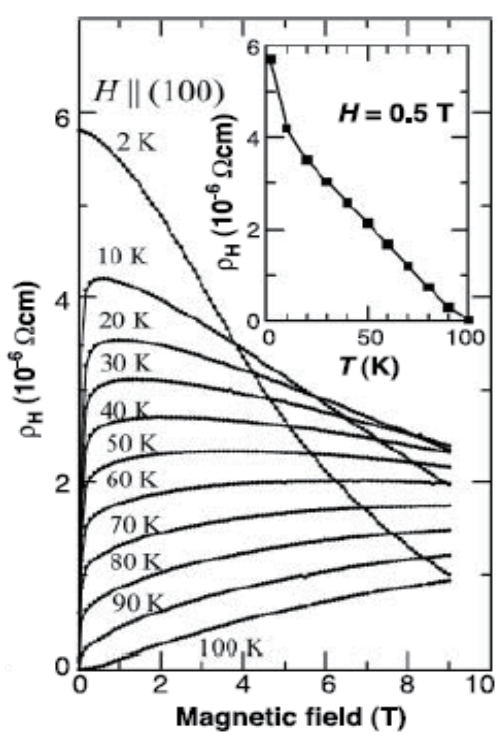

(c)

Fig.9 Anomalous Hall effect in $\mathrm{Nd}_{2 \mathrm{Mo2}} \mathrm{O}$. (a) The crystal structure contains the two inter-penetrating networks of tetrahedrons of $\mathrm{Nd}$ and Mo atoms, which are coupled antiferromagnetically. Due to the strong spin anisotropy $\mathrm{Nd}$ spins forms the non-coplanar configuration with scalar spin chirality at low temperature, which is transferred to conduction electron spins on Mo atoms as shown in (b). (c) The magnetic field dependence of the Hall resistivity for several temperature. Due to the scalar spin chirality, it shows the rapid increase as the temperature is lowered. (inset). 

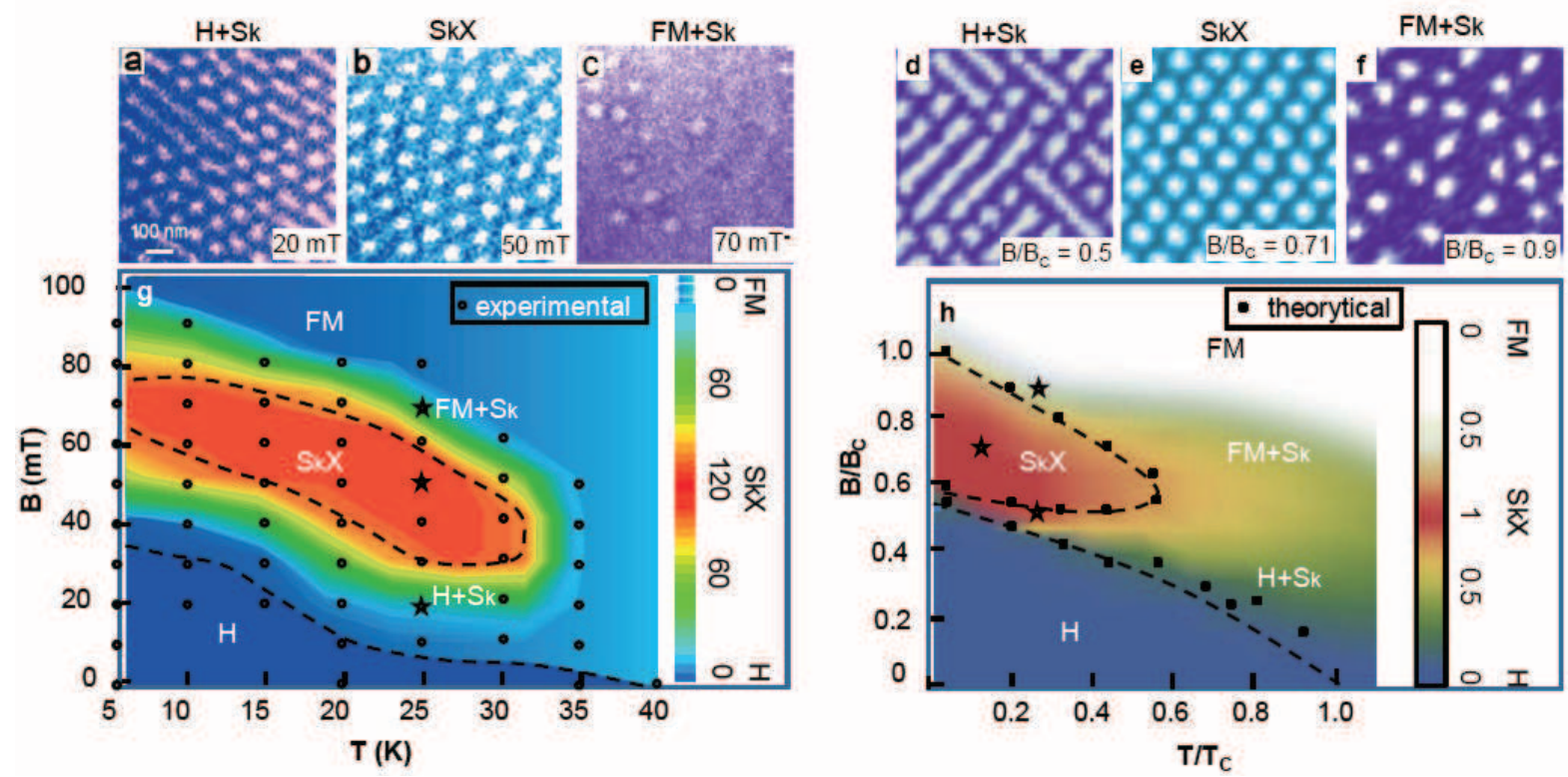

Fig.10 Skyrmions and Skyrmion crystal in (Fe,Co)Si. Left panel: experimental observation by Lorentz microscope ((a),(b),(c)) at each point shown by the star In the phase diagram (g). Right panel: the corresponding figures obtained by the Monte Carlo simulation for DM magnet in 2D. 

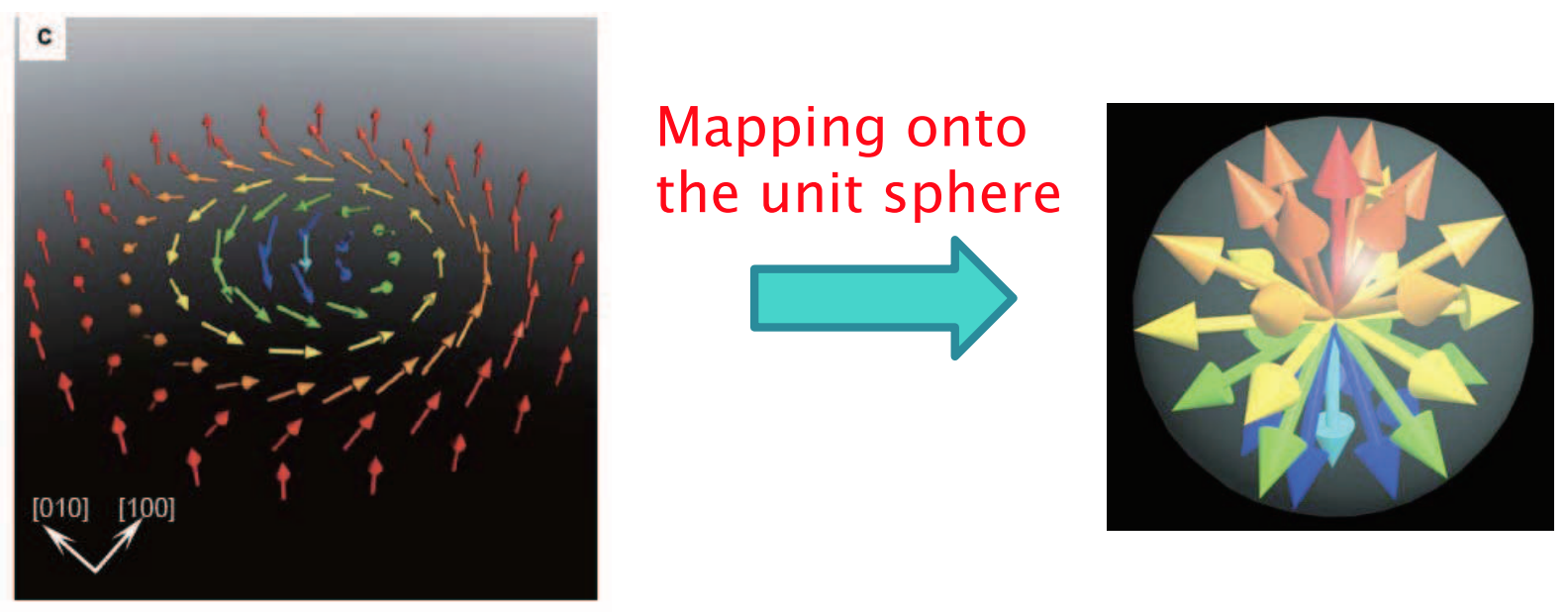

Fig.11 A single Skyrmion viewed as the mapping from the 2D real space to the unit sphere. This mapping is characterized by a topological number called Skyrmion number, i.e., how many times the mapping wrap the unit sphere. 


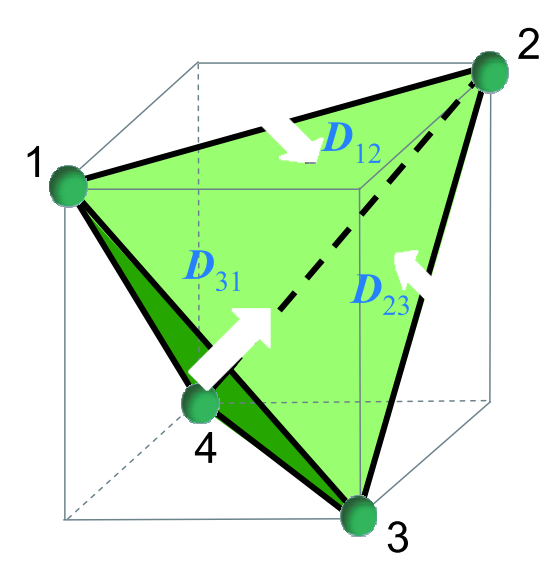

(a)

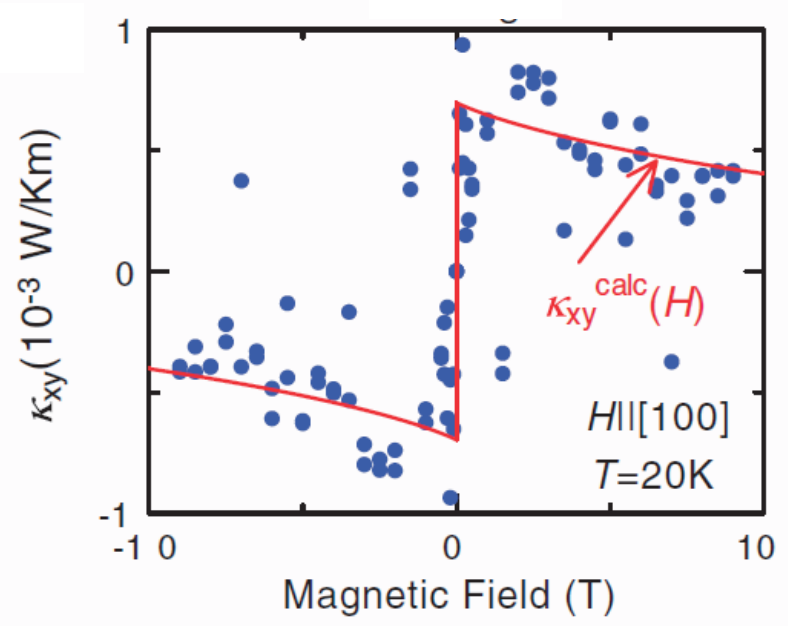

(b)

Fig.12 Thermal Hall effect in Lu2V2O7. (a) the distribution of the DM vectors for a tetrahedron. (b) the magnetic field dependence of the thermal Hall conductivity at $\mathrm{T}=20 \mathrm{~K}$ compared with the calculation (eq.(36) in the text). 